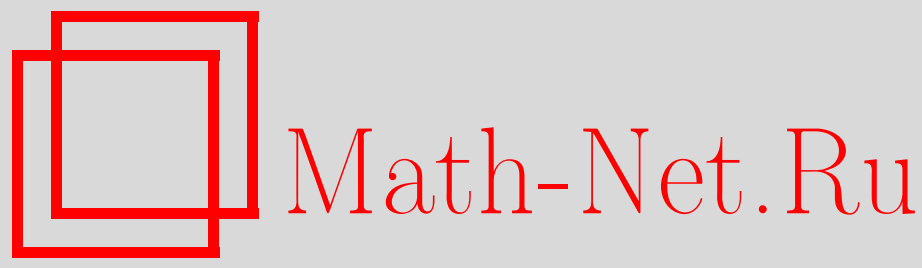

В. А. Загребнов, Х. Тамура, Модель неавтономной динамики, управляемой повторяющимся гармоническим взаимодействием, ТМФ, 2016, том 187, номер 3, 531-559

DOI: https://doi.org/10.4213/tmf9004

Использование Общероссийского математического портала Math-Net.Ru подразумевает, что вы прочитали и согласны с пользовательским соглашением http://www . mathnet.ru/rus/agreement

Параметры загрузки:

IP : 35.173 .219 .149

26 апреля 2023 г., 15:49:43

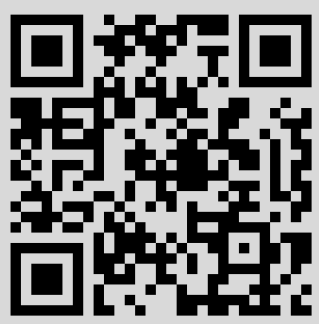




\title{
МОДЕЛЬ НЕАВТОНОМНОЙ ДИНАМИКИ, УПРАВЛЯЕМОЙ ПОВТОРЯЮЩИМСЯ ГАРМОНИЧЕСКИМ ВЗАИМОДЕЙСТВИЕМ
}

\begin{abstract}
Рассмотрена точно решаемая модель неавтономной $W^{*}$-динамики, управляющейся повторяющимся гармоническим взаимодействием. Динамика является гамильтоновой и квазисвободной. Вследствие неупругого взаимодействия в пределе больших времен происходит релаксация начальных состояний к стационарным состояниям. Выведена в явном виде скорость производства энтропии, сопровождающего эту релаксацию. Также изучается эволюция различных подсистем для прояснения корреляции событий и сходимости к равновесным состояниям. Доказано, что в пределе малого времени взаимодействия $W^{*}$-динамика проявляет универсальное стационарное поведение.
\end{abstract}

Ключевые слова: $W^{*}$-динамика, повторяющееся возмущение.

DOI: $10.4213 / \operatorname{tmf} 9004$

\section{1. ПРЕДВАРИТЕЛЬНЫЕ СВЕДЕНИЯ И МОДЕЛЬ}

1.1. Основные положения модели. Рассматриваемая модель представляет собой квантовую систему, недавно предложенную в работе [1]. Система является гармонической (одномодовый квантовый осциллятор $\mathcal{S}$ ) и последовательно возмущается зависящим от времени стационарным повторяющимся гармоническим взаимодействием. Последовательность возмущений включается в момент времени $t=0$ и далее действует на интервале $0 \leqslant t<\infty$. Обычно такая последовательность представляется как повторяющиеся взаимодействия системы $\mathcal{S}$ с бесконечной и эквидистантной по времени цепочкой $\mathcal{C}=\mathcal{S}_{1}+\mathcal{S}_{2}+\cdots$ подсистем $\left\{\mathcal{S}_{k}\right\}_{k \geqslant 1}$.

X. Тамура выражает благодарность Japan Society for the Promotion of Science за финансовую поддержку (грант (C) 24540168).

*Institut de Mathématiques de Marseille, Marseille, France

${ }^{\dagger}$ Département de Mathématiques, Université d'Aix-Marseille, Marseille, France. E-mail: Valentin.Zagrebnov@univ-amu.fr

${ }^{\ddagger}$ Institute of Science and Engineering, Graduate School of the Natural Science and Technology, Kanazawa University, Kanazawa, Japan. E-mail: tamurah@staff.kanazawa-u.ac.jp 
Мы не будем обсуждать физическую интерпретацию, стоящую за нашими математическими построениями, и отсылаем читателей к работам [2], [3] и приведенной в них литературе. В модели из работы [1] система $\mathcal{C}_{N}$ отождествляется с цепочкой $N$ квантовых частиц ("атомов") с бесконечно большим числом гармонических внутренних степеней свободы. Они последовательно взаимодействуют с одномодовым квантовым резонатором $\mathcal{S}$. Это можно считать слабой имитацией системы одноатомного мазера. В отличие от модели из работы [2], но аналогично двухуровневым атомам Джейнса-Камингса [3], взаимодействие с гармоническими атомами является неупругим. Эволюция модели из работы [1] существенно отличается от эволюции модели из работы [2], где взаимодействие упругое (см. п. 3.2 и раздел 4 настоящей статьи).

Ниже мы будем предполагать, что состояния системы $\mathcal{S}$ и каждой подсистемы $\mathcal{S}_{k}$ являются нормальными, т. е. определяются матрицами плотности $\rho_{0}$ и $\rho_{k}$ в гильбертовых пространствах $\mathscr{H}_{\mathcal{S}}$ и $\mathscr{H}_{\mathcal{S}_{k}}$ соответственно, $k=1,2, \ldots$. Тогда гильбертово пространство полной системы есть тензорное произведение $\mathscr{H}_{\mathcal{S}} \otimes \mathscr{H}_{\mathcal{C}}$, где бесконечное произведение $\mathscr{H}_{\mathcal{C}}=\bigotimes_{k \geqslant 1} \mathscr{H}_{\mathcal{S}_{k}}$ обозначает цепочку гильбертовых пространств.

Поскольку в любой фиксированный момент времени $t \geqslant 0$ в динамике участвует только конечное число $N(t)$ повторяющихся взаимодействий, подсистемы $\mathcal{S}_{k}$ при различных $k>N(t)$ все еще независимы, а также не зависят от компонент $\mathcal{S}$ и $\left\{\mathcal{S}_{k}\right\}_{k=1}^{N(t)}$. Проблема корреляций между компонентами $\mathcal{S}, \mathcal{S}_{k}$ при $k \leqslant N(t)$ и между $\mathcal{S}_{k}, \mathcal{S}_{k^{\prime}}$ при $1 \leqslant k<k^{\prime} \leqslant N(t)$ рассматривается в разделе 4 . Такая особенность повторяющихся взаимодействий позволяет свести анализ динамики к рассмотрению конечного тензорного произведения $\mathscr{H}_{\mathcal{C}_{N}}=\bigotimes_{k=1}^{N} \mathscr{H}_{\mathcal{S}_{k}}$. Тогда мы можем получить упомянутую бесконечную цепочку $\mathcal{S}+\mathcal{C}$ как апостериорный предел при $N \rightarrow \infty$.

Подробное описание динамики приведено в разделе 2.

В заключение данного пункта сформулируем некоторые предположения.

ГиПотезА 1. (Начальные состояния.) При $t \leqslant 0$ все компоненты систем $\mathcal{S}$ $u\left\{\mathcal{S}_{k}\right\}_{k=1}^{N}$ являются независимыми, т.е. состояние системы $\mathcal{S}+\mathcal{C}_{N}$ описывается как конечное тензорное произведение

$$
\omega_{\mathcal{S}+\mathcal{C}_{N}}:=\omega_{\mathcal{S}} \otimes\left(\bigotimes_{k=1}^{N} \omega_{\mathcal{S}_{k}}\right) .
$$

Мъ будем предполагать, что каждое из состояний в этом произведении является нормальным.

ГипотезА 2. (Настройка взаимодействия.) Рассмотрим повторяющиеся возмущения в режиме настройки: в любой момент времени $t \geqslant 0$ в точности одна подсистема ("атом") $\mathcal{S}_{n}$ взаимодействует с системой $\mathcal{S}$ (квантовым резонатором) в течение фиксированного времени $\tau>0$. Здесь $n=[t / \tau]+1$, где $[x]$ обозначает иелую часть числа $x \geqslant 0$.

Пусть $\mathscr{H}_{0}$ - гильбертово пространство системы $\mathcal{S}$, а $\mathscr{H}_{k}$ - гильбертово пространство системы $\mathcal{S}_{k}, k=1, \ldots, N$. Тогда для каждого $k=0,1, \ldots, N$ пространство $\mathscr{H}_{k}$ является копией одномодового бозонного фоковского пространства $\mathscr{F}$ с вакуумным вектором $\Omega \in \mathscr{F}$ и с плотно определенными операторами уничтожения и рождения 
бозона $a$ и $a^{*}$ (сопряженный оператор), причем вакуумный вектор определяется равенством $a \Omega=0$. Полная система $\mathcal{S}+\mathcal{C}_{N}$ живет в гильбертовом пространстве

$$
\mathscr{H}^{(N)}:=\mathscr{H}_{0} \otimes\left(\bigotimes_{k=1}^{N} \mathscr{H}_{k}\right)=\mathscr{F}^{\otimes(N+1)}
$$

Обозначим через $\mathbb{1}$ единичный оператор в $\mathscr{F}$. В пространстве (1.1) определим операторы

$$
b_{k}:=\mathbb{1} \otimes \cdots \otimes \mathbb{1} \otimes a \otimes \mathbb{1} \otimes \cdots \otimes \mathbb{1}, \quad b_{k}^{*}:=\mathbb{1} \otimes \cdots \otimes \mathbb{1} \otimes a^{*} \otimes \mathbb{1} \otimes \cdots \otimes \mathbb{1},
$$

где операторы $a$ или $a^{*}$ являются $(k+1)$-ми множителями в произведениях. Операторы (1.2) формально удовлетворяют каноническим коммутационным соотношениям $(\mathrm{KKC})$

$$
\left[b_{k}, b_{k^{\prime}}^{*}\right]=\delta_{k, k^{\prime}} \mathbb{1}, \quad\left[b_{k}, b_{k^{\prime}}\right]=\left[b_{k}^{*}, b_{k^{\prime}}^{*}\right]=0, \quad k, k^{\prime}=0,1, \ldots, N .
$$

ГиПотеЗА 3. (Гармоническое взаимодействие.) Зависящее от времени повторяющееся взаимодействие из гипотезы 2 представляет собой кусочно-постоянный оператор в пространстве (1.1). Он равен сумме $n \geqslant 1$ билинейных форм $K_{n}(t)$ по операторам (1.2) в пространстве $\mathscr{H}_{0} \otimes \mathscr{H}_{n}$, заданных как

$$
K_{n}(t):=\chi_{[(n-1) \tau, n \tau)}(t) \eta\left(b_{0}^{*} b_{n}+b_{n}^{*} b_{0}\right), \quad \eta>0 .
$$

Здесъ $\chi_{\mathcal{I}}(x)$ - характеристическая функиия множества $\mathcal{I}$.

1.2. Модель. Для любых $N \geqslant 1$ и $t<N \tau$ самосопряженный гамильтониан $H_{N}(t)$ неавтономной системы $\mathcal{S}+\mathcal{C}_{N}$ определяется в пространстве (1.1) как сумма гамильтонианов, отвечающих системам $\mathcal{S}, \mathcal{S}_{k}$ и взаимодействию (1.4) [1]:

$$
\begin{aligned}
H_{N}(t) & :=H_{\mathcal{S}}+\sum_{k=1}^{N}\left(H_{\mathcal{S}_{k}}+K_{k}(t)\right)= \\
& =E b_{0}^{*} b_{0}+\epsilon \sum_{k=1}^{N} b_{k}^{*} b_{k}+\eta \sum_{k=1}^{N} \chi_{[(k-1) \tau, k \tau)}(t)\left(b_{0}^{*} b_{k}+b_{k}^{*} b_{0}\right) .
\end{aligned}
$$

ГиПотеЗА 4. (Полуограниченность гамильтониана.) Чтобы самосопряженный гамильтониан (1.5) оставался полуограниченным снизу, будем предполагать, что $E>0, \epsilon>0$, и наложим условие

$$
\eta^{2} \leqslant E \epsilon
$$

Согласно соотношениям $(1.4),(1.5)$ только подсистема $\mathcal{S}_{n}$ взаимодействует с $\mathcal{S}$ при $t \in[(n-1) \tau, n \tau), n \geqslant 1$, т. е. на этом временном интервале система $\mathcal{S}+\mathcal{C}_{N}$ является автономной с самосопряженным гамильтонианом

$$
H_{n}:=E b_{0}^{*} b_{0}+\epsilon \sum_{k=1}^{N} b_{k}^{*} b_{k}+\eta\left(b_{0}^{*} b_{n}+b_{n}^{*} b_{0}\right), \quad n \leqslant N
$$


Заметим, что существует сохраняющее $\mathrm{KKC} \mathrm{линейное} \mathrm{преобразование,} \mathrm{которое} \mathrm{диа-}$ гонализует оператор (1.7):

$$
\widetilde{H}_{n}:=\varepsilon_{0} c_{0}^{*} c_{0}+\varepsilon_{1} c_{1}^{*} c_{1}+\sum_{k=2}^{N} \varepsilon_{k} c_{k}^{*} c_{k} .
$$

Здесь в соответствии с гипотезой 4

$$
\begin{aligned}
& \varepsilon_{2}=\cdots=\varepsilon_{N}=\epsilon, \\
& \varepsilon_{0}:=\frac{1}{2}\left[(E+\epsilon)+\sqrt{(E-\epsilon)^{2}+4 \eta^{2}}\right]>0, \quad \varepsilon_{1}:=\frac{1}{2}\left[(E+\epsilon)-\sqrt{(E-\epsilon)^{2}+4 \eta^{2}}\right]>0 .
\end{aligned}
$$

Из гармонической структуры гамильтониана (1.7) следует ключевая лемма.

ЛЕмма 1.1. Для $j=0,1, \ldots, N$ u $n=1,2 \ldots, N$ получаем при $t \geqslant 0$ следующие равенства:

$$
\begin{array}{rlrl}
e^{i t H_{n}} b_{j} e^{-i t H_{n}} & =\sum_{k=0}^{N}\left(U_{n}^{*}(t)\right)_{j k} b_{k}, & e^{i t H_{n}} b_{j}^{*} e^{-i t H_{n}}=\sum_{k=0}^{N} \overline{\left(U_{n}^{*}(t)\right)_{j k}} b_{k}^{*}, \\
e^{-i t H_{n}} b_{j} e^{i t H_{n}}=\sum_{k=0}^{N}\left(U_{n}(t)\right)_{j k} b_{k}, & e^{-i t H_{n}} b_{j}^{*} e^{i t H_{n}}=\sum_{k=0}^{N} \overline{\left(U_{n}(t)\right)_{j k}} b_{k}^{*} .
\end{array}
$$

Здесъ $U_{n}(t)$ и $V_{n}(t)$ - матриць размера $(N+1) \times(N+1)$; элементы матрицьь $V_{n}(t)$ задаются как

$$
\left(V_{n}(t)\right)_{j k}:=\left\{\begin{array}{cl}
g(t) z(t) \delta_{k 0}+g(t) w(t) \delta_{k n} & \text { при } j=0 \\
g(t) w(t) \delta_{k 0}+g(t) z(-t) \delta_{k n} & \text { nри } j=n \\
\delta_{j k} & \text { в инъх случаях }
\end{array}\right.
$$

¿əe

$$
\begin{aligned}
& g(t):=e^{i t(E-\epsilon) / 2}, \quad w(t):=\frac{2 i \eta}{\sqrt{(E-\epsilon)^{2}+4 \eta^{2}}} \sin \left(t \sqrt{\frac{(E-\epsilon)^{2}}{4}+\eta^{2}}\right), \\
& z(t):=\cos \left(t \sqrt{\frac{(E-\epsilon)^{2}}{4}+\eta^{2}}\right)+\frac{i(E-\epsilon)}{\sqrt{(E-\epsilon)^{2}+4 \eta^{2}}} \sin \left(t \sqrt{\frac{(E-\epsilon)^{2}}{4}+\eta^{2}}\right) ;
\end{aligned}
$$

матрица $U_{n}(t)$ связана с $V_{n}(t)$ соотношением $U_{n}(t):=e^{i t \epsilon} V_{n}(t)$.

ЗАмЕчАниЕ 1.1. Согласно определениям (1.12) и (1.13) мы получаем равенства $|z(t)|^{2}+|w(t)|^{2}=1, z(-t)=\overline{z(t)}$ и $w(t)=-\overline{w(t)}$. Поэтому матрица

$$
M(t):=\left(\begin{array}{cc}
z(t) & w(t) \\
w(t) & z(-t)
\end{array}\right)
$$

является унитарной. При $N=1$ получаем $M(t)=\overline{g(t)} V_{1}(t)$ (см. формулу (1.11)). Более того, из соотношений (1.9) и (1.10) следует, что $\left\{V_{n}(t)\right\}_{t \in \mathbb{R}}$ и $\left\{U_{n}(t)\right\}_{t \in \mathbb{R}}$ в действительности являются однопараметрическими группами унитарных матриц размера $(N+1) \times(N+1)$. 
ДокАЗАТЕЛЬСТво ЛЕммы 1.1. Пусть $\left\{J_{n}\right\}_{n=1}^{N}$ и $\left\{X_{n}\right\}_{n=1}^{N}$ - эрмитовы матрицы размера $(N+1) \times(N+1)$ с элементами

$$
\begin{gathered}
\left(J_{n}\right)_{j k}:= \begin{cases}1 & \text { при } j=k=0 \text { или } j=k=n, \\
0 & \text { в иных случаях, }\end{cases} \\
\left(X_{n}\right)_{j k}:= \begin{cases}(E-\epsilon) / 2 & \text { при }(j, k)=(0,0), \\
-(E-\epsilon) / 2 & \text { при }(j, k)=(n, n), \\
\eta & \text { при }(j, k)=(0, n), \\
\eta & \text { при }(j, k)=(n, 0), \\
0 & \text { в иных случаях. }\end{cases}
\end{gathered}
$$

Определим матрицы

$$
Y_{n}:=\epsilon I+\frac{E-\epsilon}{2} J_{n}+X_{n}, \quad n=1, \ldots, N,
$$

где $I$ - единичная матрица размера $(N+1) \times(N+1)$. Тогда гамильтониан $(1.7)$ принимает вид

$$
H_{n}=\sum_{j, k=0}^{N}\left(Y_{n}\right)_{j k} b_{j}^{*} b_{k}
$$

Поскольку матрица $Y_{n}$ эрмитова, существует диагональная матрица $\Lambda$ и унитарное отображение $\mathcal{U}_{n}: \mathbb{R}^{N+1} \rightarrow \mathbb{R}^{N+1}$, такое что $Y_{n}=\mathcal{U}_{n}^{*} \Lambda \mathcal{U}_{n}$. Напомним, что после канонического преобразования матрица $\Lambda:=\left\{\Lambda_{i j}\right\}_{i, j=0}^{N}=\left\{\delta_{i j} \varepsilon_{j}\right\}_{i, j=0}^{N}$ является универсальной и не зависит от $n$.

Новые операторы

$$
c_{j}=\sum_{k=0}^{N}\left(\mathcal{U}_{n}\right)_{j k} b_{k}, \quad c_{j}^{*}=\sum_{k=0}^{N} \overline{\left(\mathcal{U}_{n}\right)_{j k}} b_{k}^{*}, \quad j=0,1, \ldots, N,
$$

удовлетворяют ККС в пространстве $\mathscr{H}^{(N)}(1.1)$ и диагонализуют оператор (1.17):

$$
\widetilde{H}_{n}=\sum_{j=0}^{N} \Lambda_{j j} c_{j}^{*} c_{j}, \quad \Lambda_{j j}=\varepsilon_{j} .
$$

Поэтому множество всех собственных векторов оператора $\widetilde{H}_{n}$ имеет вид

$$
\left\{\prod_{j=0}^{N} \frac{\left(c_{j}^{*}\right)^{n_{j}}}{\sqrt{n_{j} !}} \Omega \otimes \cdots \otimes \Omega \mid n_{j} \in \mathbb{Z}_{+}, j=0,1, \ldots, N\right\} .
$$

Заметим, что мы получили ортонормированный базис в $\mathscr{H}^{(N)}$. Линейная оболочка $\mathscr{H}_{0}^{(N)}$ множества (1.19) является инвариантным подпространством при преобразованиях $e^{i t \widetilde{H}_{n}}$, и ее замыкание совпадает с $\mathscr{H}^{(N)}$. Тогда в силу (1.18) получаем для векторов (1.19)

$$
e^{i t \widetilde{H}_{n}} c_{j} e^{-i t \widetilde{H}_{n}}=e^{-i t \Lambda_{j j}} c_{j}, \quad e^{i t \widetilde{H}_{n}} c_{j}^{*} e^{-i t \widetilde{H}_{n}}=e^{i t \Lambda_{j j}} c_{j}^{*} .
$$


Теперь с учетом канонического преобразования (1.18) имеем

$$
\begin{aligned}
& e^{i t H_{n}} b_{j} e^{-i t H_{n}}=\sum_{k=0}^{N}\left(\mathcal{U}_{n}^{*}\right)_{j k} e^{i t \widetilde{H}_{n}} c_{k} e^{-i t \widetilde{H}_{n}}= \\
& =\sum_{k, l=0}^{N}\left(\mathcal{U}_{n}^{*}\right)_{j k} e^{-i t \Lambda_{k k}}\left(\mathcal{U}_{n}\right)_{k l} b_{l}=\sum_{l=0}^{N}\left(e^{-i t \mathcal{U}_{n}^{*} \Lambda \mathcal{U}_{n}}\right)_{j l} b_{l}=\sum_{l=0}^{N}\left(e^{-i t Y_{n}}\right)_{j l} b_{l} .
\end{aligned}
$$

Аналогично получаем

$$
e^{i t H_{n}} b_{j}^{*} e^{-i t H_{n}}=\sum_{l=0}^{N} \overline{\left(e^{-i t Y_{n}}\right)_{j l}} b_{l}^{*} .
$$

Заметим, что, используя соотношения (1.14), (1.15), мы получаем тождества

$$
X_{n}^{2}=\left(\frac{(E-\epsilon)^{2}}{4}+\eta^{2}\right) J_{n}, \quad J_{n} X_{n}=X_{n}
$$

Вместе с определениями (1.16) и (1.11) это дает

$$
\begin{aligned}
e^{i t Y_{n}}= & e^{i t \epsilon}\left(I-J_{n}+e^{i t(E-\epsilon) / 2}\left\{J_{n} \cos \left(t \sqrt{\frac{(E-\epsilon)^{2}}{4}+\eta^{2}}\right)+\right.\right. \\
& \left.\left.+i X_{n}\left(\frac{(E-\epsilon)^{2}}{4}+\eta^{2}\right)^{-1 / 2} \sin \left(t \sqrt{\frac{(E-\epsilon)^{2}}{4}+\eta^{2}}\right)\right\}\right)= \\
= & e^{i t \epsilon} V_{n}(t)=U_{n}(t) .
\end{aligned}
$$

Теперь, подставляя полученное равенство в (1.20), мы доказываем (1.9). Поскольку $U_{n}(t)^{*}=U_{n}(-t)$, можно аналогично получить (1.10). Лемма доказана.

ЗАмЕчАниЕ 1.2. Здесь и далее мы используем сокращенные обозначения

$$
g:=g(\tau), \quad w:=w(\tau), \quad z:=z(\tau), \quad V_{n}:=V_{n}(\tau), \quad U_{n}:=U_{n}(\tau)
$$

В заключение этого раздела приведем краткое содержание настоящей работы. В разделе 2 дано явное описание гамильтоновой динамики для неавтономной системы $\mathcal{S}+\mathcal{C}$, управляющейся гармоническими повторяющимися взаимодействиями (см. гипотезу 3). Показано, что наша модель бозонов (1.5) представляет собой квазисвободную $W^{*}$-динамическую систему. В п. 3.1 мы напоминаем формулы для энтропии ККС квазисвободных состояний. Они используются в п. 3.2 для вычисления производства энтропии. Раздел 4 посвящен анализу редуцированной динамики подсистем, их корреляциям и сходимости к равновесному состоянию. В конце раздела мы доказываем универсальность предела короткого времени взаимодействия для этой динамики в случае подсистемы $\mathcal{S}$. 


\section{2. НЕАВТОНОМНАЯ ДИНАМИКА ЛИУВИЛЛЯ}

\section{1. Динамика и двойственная динамика. Характеристическая функ-} ция. Начнем этот раздел с анализа эволюции неавтономной системы $\mathcal{S}+\mathcal{C}$ с гамильтонианом (1.5). Поскольку математическое описание эволюции неограниченных операторов рождения-уничтожения связано с некоторыми техническими проблемами, общий прием заключается в построении динамики на соответствующей унитальной $C^{*}$-алгебре Вейля $\mathrm{KKC}[4]$. Для подсистемы $\mathcal{S}$ это $C^{*}$-алгебра $\mathrm{KKC}$ $\mathscr{A}(\mathscr{F})$, порожденная на фоковском пространстве $\mathscr{F}$ как замыкание по операторной норме линейной оболочки $\mathscr{A}_{w}$ системы операторов Вейля

$$
\left\{\hat{w}(\alpha)=e^{i \Phi(\alpha)}\right\}_{\alpha \in \mathbb{C}} .
$$

Здесь $\Phi(\alpha):=\left(\bar{\alpha} a+\alpha a^{*}\right) / \sqrt{2}-$ самосопряженный оператор с областью определения в $\mathscr{F}$. Тогда $\mathrm{KKC}$ принимают вейлевский вид

$$
\hat{w}\left(\alpha_{1}\right) \hat{w}\left(\alpha_{2}\right)=e^{-i \operatorname{Im}\left(\bar{\alpha}_{1} \alpha_{2}\right) / 2} \hat{w}\left(\alpha_{1}+\alpha_{2}\right), \quad \alpha_{1}, \alpha_{2} \in \mathbb{C} .
$$

Заметим, что $\mathscr{A}(\mathscr{F})$ является минимальной $C^{*}$-алгеброй, которая содержит линейную оболочку $\mathscr{A}_{w}$ системы операторов Вейля (2.1). Алгебра $\mathscr{A}(\mathscr{F})$ содержится в унитальной $C^{*}$-алгебре $\mathcal{L}(\mathscr{F})$ всех ограниченных операторов на $\mathscr{F}$.

Аналогично определим $C^{*}$-алгебру Вейля $\mathscr{A}(\mathscr{H}) \subset \mathcal{L}(\mathscr{H})$ над $\mathscr{H}:=\mathscr{H}^{(N)}(1.1)$, подходящую для описания системы $\mathcal{S}+\mathcal{C}$. Эта алгебра порождается операторами

$$
W(\zeta)=\bigotimes_{k=0}^{N} \hat{w}\left(\zeta_{k}\right), \quad \zeta=\left\{\zeta_{k}\right\}_{k=0}^{N} \in \mathbb{C}^{N+1}, \quad N \geqslant 1
$$

Используя определения бозонных операторов $\left\{b_{k}, b_{k}^{*}\right\}_{k=1}^{N}$ и полуторалинейных форм

$$
\langle\zeta, b\rangle:=\sum_{j=0}^{N} \bar{\zeta}_{j} b_{j}, \quad\langle b, \zeta\rangle:=\sum_{j=0}^{N} \zeta_{j} b_{j}^{*}
$$

можно переписать операторы Вейля (2.3) в виде

$$
W(\zeta)=\exp \left[i \frac{\langle\zeta, b\rangle+\langle b, \zeta\rangle}{\sqrt{2}}\right]
$$

Обозначим через $\mathfrak{C}_{1}(\mathscr{F}) \subset \mathcal{L}(\mathscr{F})$ множество всех ядерных операторов на $\mathscr{F}$. Самосопряженный неотрицательный оператор $\rho \in \mathfrak{C}_{1}(\mathscr{F})$ с единичным следом называется матрищей плотности. Нормалънъм является состояние $\omega_{\rho}(\cdot)$, порожденное на $C^{*}$-алгебре ограниченных операторов $\mathcal{L}(\mathscr{F})$ оператором $\rho$ как

$$
\omega_{\rho}(A):=\operatorname{Tr}_{\mathscr{F}}(\rho A), \quad A \in \mathcal{L}(\mathscr{F}) .
$$

Пусть $\left\{\rho_{k}\right\}_{k=0}^{N}-$ матрицы плотности на $\mathscr{F}$. Нормальное состояние-произведение на $C^{*}$-алгебре $\mathscr{A}(\mathscr{H})$ (изометрически изоморфное тензорному произведению $\left.\bigotimes_{k=0}^{N} \mathscr{A}(\mathscr{F})\right)$ задается как

$$
\omega_{\rho}(\cdot):=\operatorname{Tr}_{\mathscr{H}}\left(\rho^{\otimes} \cdot\right), \quad \rho^{\otimes}:=\bigotimes_{k=0}^{N} \rho_{k} .
$$


Если мы положим $C_{k}(\alpha):=\operatorname{Tr}_{\mathscr{F}}\left[\rho_{k} \hat{w}(\alpha)\right]$ для $\alpha \in \mathbb{C}$, то в соответствии с $(2.3)$ получим представление для $\rho^{\otimes}$ :

$$
\omega_{\rho}(W(\zeta)):=\operatorname{Tr}_{\mathscr{H}}\left[\rho^{\otimes} W(\zeta)\right]=\prod_{k=0}^{N} C_{k}\left(\zeta_{k}\right) .
$$

Пусть $\varrho \in \mathfrak{C}_{1}(\mathscr{H})$ - матрица плотности на $\mathscr{H}$. Тогда для системы $\mathcal{S}+\mathcal{C}$ гамильтонова эволюция $T_{t}: \varrho \mapsto \varrho(t)$ начальной матрицы плотности $\varrho(0):=\varrho$ определяется как решение задачи Коши для неавтономного уравнения Лиувилля

$$
\partial_{t} \varrho(t)=L(t)(\varrho(t)),\left.\quad \varrho(t)\right|_{t=0}=\varrho .
$$

Согласно соотношению (1.7) это уравнение является автономным для каждого интервала $[(n-1) \tau, n \tau)$ :

$$
L(t)(\cdot)=L_{n}(\cdot)=-i\left[H_{n}, \cdot\right], \quad t \in[(n-1) \tau, n \tau), \quad n \geqslant 1,
$$

т. е. генератор Лиувилля кусочно не зависит от времени.

Поскольку любое значение $t \geqslant 0$ может быть представлено в виде

$$
t:=n(t) \tau+\nu(t), \quad n(t):=[t / \tau], \quad \nu(t) \in[0, \tau),
$$

непрерывное по следовой норме $\|\cdot\|_{1}$ решение задачи Коши (2.9) [5] можно найти с помощью итераций:

$$
\begin{aligned}
\varrho(t) & =T_{t}(\varrho):=T_{\nu(t), n}\left(T_{\tau, n-1}\left(\ldots T_{\tau, 1}(\varrho) \ldots\right)\right)= \\
& =e^{-i \nu(t) H_{n}} e^{-i \tau H_{n-1}} \ldots e^{-i \tau H_{1}} \varrho e^{i \tau H_{1}} \ldots e^{i \tau H_{n-1}} e^{i \nu(t) H_{n}} .
\end{aligned}
$$

Здесь $t \in[(n-1) \tau, n \tau)$ и $n=n(t)<N$. Вследствие непрерывности по норме $\|\cdot\|_{1}$ из $(2.12)$ получаем

$$
\varrho(N \tau-0)=\varrho(N \tau)=T_{N \tau}(\varrho)=e^{-i \tau H_{N}} \ldots e^{-i \tau H_{1}} \varrho e^{i \tau H_{1}} \ldots e^{i \tau H_{N}} .
$$

ЗАмечАНИЕ 2.1. Отметим, что

$$
U(t)=e^{-i \tau H_{1}} \ldots e^{-i \tau H_{n-1}} e^{-i \nu(t) H_{n(t)}}, \quad t=n(t) \tau+\nu(t),
$$

является однопараметрическим сильно непрерывным семейством унитарных операторов на $\mathscr{H}$. Тогда равенство $\varrho(t)=U(t) \varrho U^{*}(t)$ влечет, что при $t \in \mathbb{R}$ отображение $T_{t}(2.12)$ вполне положительно и сохраняет след, так что отображение $t \mapsto \varrho(t)$ оказывается непрерывным в слабой операторной топологии. Поскольку $\|\varrho(t)\|_{1}=1$, из слабой непрерывности следует $\|\cdot\|_{1}$-непрерывность отображения $t \mapsto \varrho(t)$ (см., например, книгу [5], следствие 2.66). Таким образом, отображение $T_{t}$ является непрерывным по следовой норме *-автоморфизмом множества

$$
\left\{\varrho \in \mathfrak{C}_{1}(\mathscr{F}): \varrho \geqslant 0,\|\varrho\|_{1}=1\right\}
$$

всех матриц плотности. 
Напомним, что эквивалентным и часто более удобным описанием эволюции матриц плотности (2.12) является двойственное отображение $T_{t}^{*}: \mathcal{L}(\mathscr{H}) \rightarrow \mathcal{L}(\mathscr{H})$, задающееся как

$$
\omega_{\varrho(t)}(A)=\operatorname{Tr}_{\mathscr{H}}\left(T_{t}(\varrho) A\right)=: \operatorname{Tr}_{\mathscr{H}}\left(\varrho T_{t}^{*}(A)\right), \quad(\varrho, A) \in \mathfrak{C}_{1}(\mathscr{F}) \times \mathcal{L}(\mathscr{H}) .
$$

Поскольку отображение $t \mapsto T_{t}(\varrho)$ является $\|\cdot\|_{1}$-непрерывным и поскольку $\mathcal{L}(\mathscr{H})$ топологически двойственно к $\mathfrak{C}_{1}(\mathscr{H})$, получаем, что $t \mapsto T_{t}^{*}(A)$ из $(2.14)$ представляет собой однопараметрический $*$-автоморфизм унитальной $C^{*}$-алгебры ограниченных операторов $\mathcal{L}(\mathscr{H})$. Автоморфизм $C^{*}$-динамической системы $\left(\mathcal{L}(\mathscr{H}), T_{t}^{*}\right)$ не является непрерывным по времени для бозонов. Чтобы обеспечить непрерывность отображения $T_{t}^{*}$, рассмотрим вместо $C^{*}$-алгебры $\mathscr{A}(\mathscr{H}) \subset \mathcal{L}(\mathscr{H})$ алгебру фон Неймана $\mathfrak{M}(\mathscr{H})$ - замыкание вейлевской линейной оболочки $\mathscr{A}_{w}$, порожденной операторами $(2.1),(2.3)$, в слабой* топологии. Поскольку эта топология слабее, чем топология $C^{*}$-алгебры, алгебра $\mathfrak{M}(\mathscr{H})$ оказывается $*$-изоморфной $\mathcal{L}(\mathscr{H})$. Тогда из $\|\cdot\|_{1}$ непрерывности $T_{t}(\varrho)$ следует непрерывность двойственного отображения $t \mapsto T_{t}^{*}(A)$ в слабой* топологии на $\mathfrak{M}(\mathscr{H})$, а также определяется $W^{*}$-динамическая система $\left(\mathfrak{M}(\mathscr{H}), T_{t}^{*}\right)$ (см., например, книгу [6]).

Ниже мы покажем, что $T_{t}^{*}$ отображает $\mathscr{A}(\mathscr{H})$ саму в себя и что действие $T_{t}^{*}$ на операторы Вейля можно вычислить в явном виде. Поскольку $\mathscr{A}(\mathscr{H})$ является слабо* плотной в $\mathcal{L}(\mathscr{H})$, можно вывести свойства эволюции $t \mapsto \rho(t)$ для нормального состояния $\omega_{\rho(t)}(\cdot)$.

Будем использовать характеристическую функиию $E_{\omega}(\zeta)$ состояния $\omega$ на $C^{*}$ алгебре Вейля $\mathscr{A}(\mathscr{H})$ :

$$
\zeta \mapsto E_{\omega}(\zeta):=\omega(W(\zeta)), \quad \zeta \in \mathbb{C}^{N+1} .
$$

Конечные линейные комбинациии операторов (2.3) плотны по норме в $\mathscr{A}(\mathscr{H})$, поэтому отображение $\zeta \mapsto W(\zeta)$ является непрерывным в сильной операторной топологии. Если функция $\mathbb{R} \ni s \mapsto E_{\omega}(s \zeta)$ непрерывна, то состояние $\omega$ регулярно.

Согласно теореме Араки-Сигала (см., например, [6]) любое состояние $\omega$ на $\mathscr{A}(\mathscr{H})$ полностью определяется своей характеристической функцией. Ниже мы будем использовать связь между сходимостью характеристических функций и состояний.

ЗАмечАниЕ 2.2. Множество состояний $S_{\mathcal{A}}$ над алгеброй $\mathscr{A}(\mathscr{H})$ является подмножеством в алгебре, двойственной к $\mathscr{A}(\mathscr{H})$, т. е. $S_{\mathcal{A}} \subset \mathscr{A}(\mathscr{H})^{*}$. Кроме равномерной топологии на $\mathscr{A}(\mathscr{H})^{*}$ рассматривается также слабая* топология. Сужение этой топологии на $S_{\mathcal{A}}$ определяется системой окрестностей

$$
\mathcal{N}\left(\omega ; A_{1}, \ldots, A_{n}\right):=\left\{\omega^{\prime} \in \mathscr{A}(\mathscr{H})^{*}:\left|\omega^{\prime}\left(A_{i}\right)-\omega\left(A_{i}\right)\right|<\varepsilon, i=1,2 \ldots, n\right\}
$$

для любого $\varepsilon>0$ и конечного набора операторов $A_{1}, A_{2}, \ldots, A_{n} \in \mathscr{A}(\mathscr{H})$. Если последовательность регулярных состояний $\left\{\omega^{(k)}\right\}_{k \geqslant 1}$ доставляет сходимость характеристических функций,

$$
\lim _{k \rightarrow \infty} E_{\omega(k)}(\zeta)=E_{\infty}(\zeta), \quad \zeta \in \mathbb{C}^{N+1},
$$

то $E_{\infty}(\zeta)$ удовлетворяет условиям теоремы Араки-Сигала и определяет на $\mathscr{A}(\mathscr{H})$ регулярное состояние $\omega^{(\infty)}$ с $E_{\omega(\infty)}(\zeta)=E_{\infty}(\zeta)$. Более того, из (2.17) следует, 
что $\omega^{(\infty)}$ есть предел последовательности $\left\{\omega^{(k)}\right\}_{k \geqslant 1}$ в слабой* топологии $(2.16)$ на $S_{\mathcal{A}}$ :

$$
\omega^{(\infty)}=\mathrm{w}^{*}-\lim _{k \rightarrow \infty} \omega^{(k)}
$$

(см. сборник [6] и книгу [4], глава 5.2.3).

2.2. Квазисвободная эволюция. Используя соотношение (2.13) и двойственное представление (2.14), получаем основной результат данного раздела. Начнем с леммы.

ЛЕмма 2.1. Характеристическая функиия $E_{\omega_{\varrho(t)}}(\zeta)$ (2.15) nри $t=N \tau$ имеет вид

$$
\omega_{\varrho(N \tau)}(W(\zeta))=\omega_{\varrho}\left(W\left(U_{1} \ldots U_{N} \zeta\right)\right)=\prod_{k=0}^{N} C_{k}\left(\left(U_{1} \ldots U_{N} \zeta\right)_{k}\right)
$$

если при $t=0$ начальное условие $\varrho:=\bigotimes_{k=0}^{N} \rho_{k}($ см. задачу Коши (2.7)). Здесь

$$
\left(U_{1} \ldots U_{N} \zeta\right)_{0}=e^{i N \tau \epsilon}\left((g z)^{N} \zeta_{0}+\sum_{j=1}^{N} g w(g z)^{j-1} \zeta_{j}\right)
$$

при этом для $0<k<N$

$$
\left(U_{1} \ldots U_{N} \zeta\right)_{k}=e^{i N \tau \epsilon}\left(g w(g z)^{N-k} \zeta_{0}+g \bar{z} \zeta_{k}+\sum_{j=k+1}^{N} g^{2} w^{2}(g z)^{j-k-1} \zeta_{j}\right)
$$

$u$

$$
\left(U_{1} \ldots U_{N} \zeta\right)_{N}=e^{i N \tau \epsilon}\left(g w \zeta_{0}+g \bar{z} \zeta_{N}\right)
$$

(см. определения (1.12) и (1.13)).

ДокАЗАтЕЛьСтво. Заметим, что формулы (2.8), (2.13) и двойственность (2.14) дают

$$
\begin{aligned}
\omega_{\varrho(N \tau)}(W(\zeta)) & =\operatorname{Tr}_{\mathscr{H}}\left[\varrho T_{N \tau}^{*}(W(\zeta))\right]=\operatorname{Tr}_{\mathscr{H}}\left[\varrho e^{i \tau H_{1}} \ldots e^{i \tau H_{N}} W(\zeta) e^{-i \tau H_{N}} \ldots e^{-i \tau H_{1}}\right]= \\
& =\operatorname{Tr}_{\mathscr{H}}\left[\varrho W\left(U_{1} \ldots U_{N} \zeta\right)\right]=\prod_{k=0}^{N} C_{k}\left(\left(U_{1} \ldots U_{N} \zeta\right)_{k}\right) .
\end{aligned}
$$

Чтобы ввести отображение $\zeta \mapsto U_{1} \ldots U_{N} \zeta$ из $(2.22)$, используем лемму 1.1 и полуторалинейные формы (2.4). Получим

$$
e^{i \tau H_{1}} \ldots e^{i \tau H_{N}}\langle\zeta, b\rangle e^{-i \tau H_{N}} \ldots e^{-i \tau H_{1}}=\left\langle\zeta, U_{N}^{*} \ldots U_{1}^{*} b\right\rangle=\left\langle U_{1} \ldots U_{N} \zeta, b\right\rangle
$$

и аналогичное выражение для сопряженного отображения, которое затем подставим в равенство (2.5).

Более того, согласно той же лемме 1.1 получаем $U_{1} \ldots U_{N} \zeta=e^{i N \tau \epsilon} V_{1} \ldots V_{N} \zeta$, где

$$
\left(V_{1} \ldots V_{N}\right)_{0 j}= \begin{cases}\left(V_{1}\right)_{00} \ldots\left(V_{N}\right)_{00}=(g z)^{N}, & j=0 \\ \left(V_{1}\right)_{00} \ldots\left(V_{j-1}\right)_{00}\left(V_{j}\right)_{0 j}\left(V_{j+1}\right)_{j j} \ldots\left(V_{N}\right)_{j j}=(g z)^{j-1} g w, & 0<j \leqslant N\end{cases}
$$


и при $0<k \leqslant N$

$$
\left(V_{1} \ldots V_{N}\right)_{k j}= \begin{cases}\left(V_{1} \ldots V_{k-1}\right)_{k k}\left(V_{k}\right)_{k 0}\left(V_{k+1} \ldots V_{N}\right)_{00}=g w(g z)^{N-k}, & j=0, \\ 0, & 0<j<k, \\ \left(V_{1} \ldots V_{k-1}\right)_{k k}\left(V_{k}\right)_{k k}\left(V_{k+1} \ldots V_{N}\right)_{k k}=g \bar{z}, & j=k, \\ \left(V_{1} \ldots V_{k-1}\right)_{k k}\left(V_{k}\right)_{k 0}\left(V_{k+1} \ldots V_{j-1}\right)_{00}\left(V_{j}\right)_{0 j}\left(V_{j+1} \ldots V_{N}\right)_{j j}= & k<j \leqslant N .\end{cases}
$$

Собирая эти формулы, получаем явные выражения для компонент (2.19) и (2.20) вектора $U_{1} \ldots U_{N} \zeta$. Лемма доказана.

ЗАмечАние 2.3. Для фиксированного значения $N$ и для любого момента времени $t=m \tau, 1 \leqslant m \leqslant N$, рассуждения леммы 2.1 дают для характеристической функции $E_{\omega_{\varrho(m \tau)}}(\zeta)(2.15)$ общую формулу

$$
\begin{aligned}
\omega_{\varrho(m \tau)}(W(\zeta)) & =\omega_{\varrho}\left(T_{m \tau}^{*}(W(\zeta))\right)= \\
& =\omega_{\varrho}\left(W\left(U_{1} \ldots U_{m} \zeta\right)\right)=\prod_{k=0}^{N} C_{k}\left(\left(U_{1} \ldots U_{m} \zeta\right)_{k}\right) .
\end{aligned}
$$

Повторяя те же рассуждения, что и для (2.20), получаем явные формулы для компонент $\left\{\left(U_{1} \ldots U_{m} \zeta\right)_{k}\right\}_{k=0}^{N}$ :

$$
\begin{aligned}
& \left(U_{1} \ldots U_{m} \zeta\right)_{k}= \\
& \quad= \begin{cases}e^{i m \tau \epsilon}\left((g z)^{m} \zeta_{0}+\sum_{j=1}^{m} g w(g z)^{j-1} \zeta_{j}\right), & k=0, \\
e^{i m \tau \epsilon}\left(g w(g z)^{m-k} \zeta_{0}+g \bar{z} \zeta_{k}+\sum_{j=k+1}^{m} g^{2} w^{2}(g z)^{j-k-1} \zeta_{j}\right), & 1 \leqslant k<m, \\
e^{i m \tau \epsilon}\left(g w \zeta_{0}+g \bar{z} \zeta_{m}\right), & k=m \\
e^{i m \tau \epsilon} \zeta_{k}, & m<k \leqslant N,\end{cases}
\end{aligned}
$$

Заметим, что при $m=N$ эти формулы совпадают с (2.19)-(2.21) за исключением последней строки, которая в данном случае является пустой.

ОПРЕДЕЛЕНиЕ 2.1. Сохраняющая единицу $*$-динамика $t \mapsto T_{t}^{*}$ на алгебре фон Неймана $\mathfrak{M}(\mathscr{H})$, порожденной операторами $\{W(\zeta)\}_{\zeta \in \mathbb{C}}(2.5)$, является квазисвободной, если существует такое отображение $U_{t}: \zeta \mapsto U_{t} \zeta$ и такая комплекснозначная функция $\Omega_{t}: \zeta \mapsto \Omega_{t}(\zeta)$, что

$$
T_{t}^{*}(W(\zeta))=\Omega_{t}(\zeta) W\left(U_{t} \zeta\right), \quad \Omega_{0}=1, \quad U_{0}=I
$$

(см., например, [6], [4] или [7]).

Теперь согласно замечанию 2.3 мы немедленно получаем основной результат для $W^{*}$-динамической системы $\left(\mathfrak{M}(\mathscr{H}), T_{t}^{*}\right)$ в рамках нашей модели.

ТЕОрема 2.1. Пошаговая двойственная динамика

$$
T_{m \tau}^{*}(W(\zeta))=W\left(U_{1} \ldots U_{m} \zeta\right), \quad m=0,1, \ldots, N
$$

является квазисвободной (см. соотношение (2.25)) при $\Omega_{t}(\zeta)=1$ и для матрии, $\left\{U_{j}\right\}_{j=1}^{N}$ на $\mathbb{C}^{N+1}$, которье определяются леммой 1.1 . 


\section{3. ЭВОЛЮЦИЯ СОСТОЯНИЙ И ПРОИЗВОДСТВО ЭНТРОПИИ}

3.1. Энтропия квазисвободных состояний. В данном пункте мы получим некоторые полезные формулы, связывающие математические ожидания операторов Вейля (вейлевские характеристические функции) и энтропию бозонных квазисвободных состояний. Полученные формулировки являются ограниченными, но достаточными для наших целей. Общие положения рассмотрены, например, в трудах [4], [6]-[8] и приведенной в них литературе.

ОПРедЕЛЕНИе 3.1. Состояние $\omega$ на $C^{*}$-алгебре $\mathrm{KKC} \mathscr{A}(\mathscr{F})(2.1)$ называется $\kappa в a$ зисвободным, если

$$
\omega_{\mathrm{T}}\left(\Phi\left(\alpha_{1}\right), \Phi\left(\alpha_{2}\right), \ldots, \Phi\left(\alpha_{m}\right)\right)=0 \quad \text { при } m \geqslant 3, \alpha_{1}, \alpha_{2}, \ldots, \alpha_{m} \in \mathbb{C} .
$$

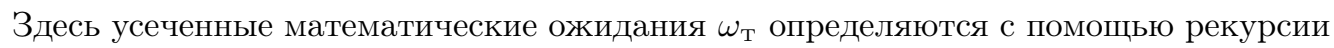

$$
\begin{aligned}
\omega_{\mathrm{T}}(\Phi(\alpha)) & :=\omega(\Phi(\alpha)), \\
\omega_{\mathrm{T}}\left(\Phi\left(\alpha_{1}\right) \Phi\left(\alpha_{2}\right)\right) & :=\omega\left(\Phi\left(\alpha_{1}\right) \Phi\left(\alpha_{2}\right)\right)-\omega\left(\Phi\left(\alpha_{1}\right)\right) \omega\left(\Phi\left(\alpha_{2}\right)\right), \quad \ldots .
\end{aligned}
$$

Таким образом, данное состояние полностью определяется линейным функционалом $\omega(\Phi(\alpha))$ и положительным билинейным функционалом

$$
\omega\left(\Phi\left(s_{1} \alpha_{1}\right) \Phi\left(s_{2} \alpha_{2}\right)\right)=s_{1} s_{2} \omega\left(\Phi\left(\alpha_{1}\right) \Phi\left(\alpha_{2}\right)\right), \quad s_{1}, s_{2} \in \mathbb{R} .
$$

Заметим, что квазисвободное состояние определяется характеристической функцией

$$
\omega(\hat{w}(\alpha)):=e^{i \omega(\Phi(\alpha))-\omega_{\mathrm{T}}\left(\Phi(\alpha)^{2}\right) / 2}, \quad \alpha \in \mathbb{C} .
$$

Тогда $C^{\infty}$-непрерывную функцию $s \mapsto \omega(\hat{w}(s \alpha)), s \in \mathbb{R}$, можно продолжить до аналитической в открытой полосе вокруг вещественной оси, что позволяет проверить

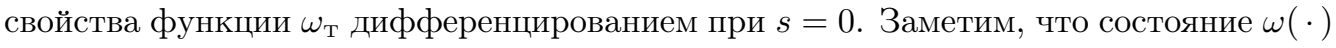
называется аналитическим, если его характеристическая функция $\omega(\hat{w}(z \alpha))$ является аналитической при $z \in \mathbb{C}$ в открытой окрестности начала координат. Поэтому любое квазисвободное состояние аналитическое.

Напомним, что квазисвободное состояние $\omega$ является калибровочно-инвариантным, если $\omega(\hat{w}(\alpha))=\omega\left(\hat{w}\left(e^{i \varphi} \alpha\right)\right)$ при $\varphi \in[0,2 \pi)$. Используя аналитичность, находим, что $\omega(\Phi(\alpha))=0$ и что состояние определяется положительной полуторалинейной формой $\mathbb{C} \times \mathbb{C} \rightarrow \omega\left(\Phi\left(\alpha_{1}\right) \Phi\left(\alpha_{2}\right)\right)$. Тогда из (3.1) и определения оператора $\Phi(\alpha)$ получаем для характеристической функции квазисвободного калибровочно-инвариантного состояния над $C^{*}$-алгеброй Фока $\mathrm{KKC}$ следующее равенство:

$$
\omega(\hat{w}(\alpha))=e^{-|\alpha|^{2} / 4-h(\alpha) / 2}, \quad \alpha \in \mathbb{C},
$$

где полуторалинейная форма есть $h(\alpha):=|\alpha|^{2} \omega\left(a^{*} a\right)$.

Пусть $\omega_{\beta}$ - гиббсовское состояние с параметром $\beta$ (безразмерная обратная температура), которое задается матрицей плотности $\rho(\beta)=e^{-\beta a^{*} a} / Z(\beta)$, где $Z(\beta)=$ $\left(1-e^{-\beta}\right)^{-1}$. Поскольку

$$
\omega_{\beta}(\hat{w}(\alpha))=e^{-|\alpha|^{2} / 4-h_{\beta}(\alpha) / 2}, \quad h_{\beta}(\alpha)=\frac{|\alpha|^{2}}{e^{\beta}-1}, \quad \alpha \in \mathbb{C},
$$


это состояние является квазисвободным и калибровочно-инвариантным. Заметим, что энтропия состояния $\omega_{\beta}$ имеет вид

$$
s(\beta):=-\operatorname{Tr}_{\mathscr{F}}[\rho(\beta) \ln \rho(\beta)]=\beta \omega_{\beta}\left(a^{*} a\right)-\ln \left(1-e^{-\beta}\right), \quad \omega_{\beta}\left(a^{*} a\right)=\frac{1}{e^{\beta}-1} .
$$

В терминах переменной $x:=\left(1+e^{-\beta}\right) /\left(1-e^{-\beta}\right)$ энтропия (3.4) равна

$$
s(\beta)=\sigma(x):=\frac{x+1}{2} \ln \frac{x+1}{2}-\frac{x-1}{2} \ln \frac{x-1}{2} .
$$

Здесь $\sigma:(1, \infty) \rightarrow(0, \infty)$ и $\sigma^{\prime}(x)>0$.

Чтобы продолжить формулу (3.5) на пространство (1.1), заметим, что общие калибровочно-инвариантные квазисвободные состояния на $C^{*}$-алгебре $\mathrm{KKC} \mathscr{A}(\mathscr{H})$ определяются матрицей плотности вида [7]

$$
\rho_{L}=\frac{1}{Z_{L}} e^{-\langle b, L b\rangle}, \quad Z_{L}=\operatorname{det}\left[I-e^{-L}\right]^{-1} .
$$

Здесь полуторалинейные операторнозначные формы $\langle b, L b\rangle=\sum_{n, m=0}^{N} \ell_{n m} b_{n}^{*} b_{m}$ параметризованы положительно-определенной эрмитовой матрицей $L=\left\{\ell_{n m}\right\}_{0 \leqslant n, m \leqslant N}$ размера $(N+1) \times(N+1)$. Заметим, что $*$-автоморфизм $G_{\varphi}$ на $\mathscr{A}(\mathscr{H})$ (калибровочное преобразование)

$$
G_{\varphi}: b_{n}^{*} \mapsto b_{n}^{*} e^{i \varphi}, \quad b_{m} \mapsto b_{m} e^{-i \varphi}, \quad \varphi \in \mathbb{R}, \quad n, m=0,1, \ldots N,
$$

оставляет состояние (3.6) инвариантным. Тогда характеристическая функция $E_{\omega_{\rho_{L}}}$ принимает вид

$$
\omega_{\rho_{L}}(W(\zeta))=\operatorname{Tr}_{\mathscr{H}}\left[\rho_{L} W(\zeta)\right]=\exp \left[-\frac{1}{4}\langle\zeta, \zeta\rangle-\frac{1}{2}\left\langle\zeta, \frac{I}{e^{L}-I} \zeta\right\rangle\right] .
$$

Здесь (транспонированный) вектор в аргументе есть $\zeta^{\operatorname{tr}}=\left(\zeta_{0}, \zeta_{1}, \ldots, \zeta_{N}\right) \in \mathbb{C}^{N+1}$. Заметим, что энтропия состояния $\omega_{\rho_{L}}$ равна

$$
S\left(\rho_{L}\right)=-\operatorname{Tr}_{\mathscr{C}}\left[\rho_{L} \ln \rho_{L}\right]=\operatorname{tr}_{\mathbb{C}^{N+1}}\left[L\left(e^{L}-I\right)^{-1}-\ln \left(I-e^{-L}\right)\right] .
$$

Если мы определим матрицу $X:=\left(I+e^{-L}\right)\left(I-e^{-L}\right)^{-1}$, то характеристическая функция (3.8) примет вид

$$
\omega_{\rho_{L}}(W(\zeta))=\exp \left[-\frac{1}{4}\langle\zeta, X \zeta\rangle\right]
$$

и для энтропии (3.9) мы получим

$$
S\left(\rho_{L}\right)=\operatorname{tr}\left[\frac{X+I}{2} \ln \frac{X+I}{2}-\frac{X-I}{2} \ln \frac{X-I}{2}\right] .
$$

Далее нам понадобятся несколько более специфические конструкции, чем те, что заданы формулами (3.10), (3.11). Пусть $\rho(\beta, \delta ; \xi)$ - матрица плотности квазисвободного состояния (3.6), соответствующего операторнозначной полуторалинейной форме

$$
\langle b, L(\beta, \delta ; \xi) b\rangle:=\beta \sum_{n=0}^{N} b_{n}^{*} b_{n}+\delta\langle b, \xi\rangle\langle\xi, b\rangle
$$

на $\mathbb{C}^{N+1} \times \mathbb{C}^{N+1}$. Здесь $\beta>0, \delta>-\beta$ и $\xi^{\operatorname{tr}}=\left(\xi_{0}, \xi_{1}, \ldots, \xi_{N}\right) \in \mathbb{C}^{N+1}$. 
ЛЕмма 3.1. Функиия распределения состояния

$$
\rho(\beta, \delta ; \xi)=\frac{1}{Z(\beta, \delta ; \xi)} e^{-\langle b, L(\beta, \delta ; \xi) b\rangle}
$$

имеет вид

$$
Z(\beta, \delta ; \xi)=\operatorname{Tr}_{\mathscr{H}}\left[e^{-\langle b, L(\beta, \delta ; \xi) b\rangle}\right]=\left(1-e^{-\beta}\right)^{-N}\left(1-e^{-(\beta+\delta\langle\xi, \xi\rangle)}\right)^{-1} .
$$

Характеристическая функиия (2.15) и энтропия этого состояния равнь

$$
\begin{aligned}
\operatorname{Tr}_{\mathscr{H}}[\rho(\beta, \delta ; \xi) W(\zeta)]= & \exp \left[-\frac{1+e^{-\beta}}{4\left(1-e^{-\beta}\right)}\langle\zeta, \zeta\rangle\right] \times \\
& \times \exp \left[-\frac{1}{4}\left(\frac{1+e^{-\beta-\delta\langle\xi, \xi\rangle}}{1-e^{-\beta-\delta\langle\xi, \xi\rangle}}-\frac{1+e^{-\beta}}{1-e^{-\beta}}\right) \frac{|\langle\xi, \zeta\rangle|^{2}}{\langle\xi, \xi\rangle}\right]
\end{aligned}
$$

$u$

$$
S(\rho(\beta, \delta ; \xi))=-\operatorname{Tr}_{\mathscr{H}}[\rho(\beta, \delta ; \xi) \ln \rho(\beta, \delta ; \xi)]=N s(\beta)+s(\beta+\delta\langle\xi, \xi\rangle) .
$$

ДоказАтельство. Равенство (3.13) следует из (3.6) и (3.12). Действительно, согласно (3.6) любое ортогональное преобразование $\mathcal{O}$ на $\mathbb{C}^{N+1}$ оставляет функцию распределения инвариантной, $Z_{\mathcal{O}^{\mathrm{T}} L \mathcal{O}}=Z_{L}$, следовательно, можно вычислить ее значение на векторе $O \xi$ (вместо $\xi$ ), где $\mathcal{O} \xi$ имеет только одну ненулевую компоненту, равную векторной норме $\langle\xi, \xi\rangle^{1 / 2}$. При таком выборе $\mathcal{O} \xi$ правая часть равенства (3.13) получается немедленно из расчета левой части.

Данное преобразование $\mathcal{O}$ также диагонализует матрицу $L:=L(\beta, \delta ; \xi)$, поэтому его используют, чтобы упростить функцию (3.10), а затем на последнем шаге вернуться к $\xi$. Для этого заметим, что

$$
\begin{aligned}
\omega_{\rho_{L}}(W(\zeta)) & =\exp \left[-\frac{1}{4}\left\langle\mathcal{O} \zeta, \mathcal{O} X \mathcal{O}^{*} \mathcal{O} \zeta\right\rangle\right]= \\
& =\exp \left[-\frac{1+e^{-\beta}}{4\left(1-e^{-\beta}\right)}\langle\mathcal{O} \zeta, \mathcal{O} \zeta\rangle^{\prime}\right] \exp \left[-\frac{1+e^{-\beta-\delta\langle\xi, \xi\rangle}}{4\left(1-e^{-\beta-\delta\langle\xi, \xi\rangle}\right)}\left|(\mathcal{O} \zeta)_{0}\right|^{2}\right] .
\end{aligned}
$$

Здесь $\langle\mathcal{O} \zeta, \mathcal{O} \zeta\rangle^{\prime}:=\sum_{k=1}^{N}\left|(\mathcal{O} \zeta)_{k}\right|^{2}$ и мы выбрали преобразование $\mathcal{O}$ таким образом, что $(\mathcal{O} \xi)_{j}=\delta_{0, j}\|\xi\|$. Поскольку

$$
\left|(\mathcal{O} \zeta)_{0}\right|^{2}=\frac{1}{\langle\xi, \xi\rangle}\langle\mathcal{O} \zeta, \mathcal{O} \xi\rangle\langle\mathcal{O} \xi, \mathcal{O} \zeta\rangle
$$

тождества (3.16) доказывают (3.14). Аналогичный метод можно использовать для энтропии (3.9). Вычисление следа в диагональном представлении для $L=L(\beta, \delta ; \xi)$ дает формулу (3.15). Лемма доказана.

Поскольку согласно теореме Араки-Сигала состояние полностью определяется своей характеристической функцией, соотношения (3.14) и (3.15) приводят к следующему утверждению.

ЛЕмма 3.2. Энтропия $S(\rho)$ квазисвободного состояния $\omega_{\rho}$ на $C^{*}$-алгебре $K К С$ $\mathscr{A}(\mathscr{H})$ с характеристической функиией

$$
\omega_{\rho}(W(\zeta))=\exp \left[-\frac{1}{4}\left(x\langle\zeta, \zeta\rangle+x_{0}|\langle\xi, \zeta\rangle|^{2}\right)\right]
$$


однозначно определяется параметрами $\left(\xi, x, x_{0}\right)$, где $\xi \in \mathbb{C}^{N+1}, x>1, x_{0}>1-x$, u имеет вид

$$
S(\rho)=N \sigma(x)+\sigma\left(x+x_{0}\langle\xi, \xi\rangle\right)
$$

где $\sigma(\cdot)$ задана в (3.5).

ДокАзАтЕльство. Утверждение леммы следует непосредственно из определения (3.5), если положить в (3.14)

$$
x_{0}\langle\xi, \xi\rangle=\frac{1+e^{-\beta-\delta\langle\xi, \xi\rangle}}{1-e^{-\beta-\delta\langle\xi, \xi\rangle}}-\frac{1+e^{-\beta}}{1-e^{-\beta}}
$$

и использовать соотношение (3.5) в (3.15).

3.2. Производство относительной энтропии. Рассмотрим эволюцию (2.12) нашей системы $\mathcal{S}+\mathcal{C}$ в случае, когда начальная матрица плотности (2.7) соответствует произведению калибровочно-инвариантных квазисвободных гиббсовских состояний с параметром $\beta_{0} \geqslant 0$ для $\mathcal{S}$ и с параметром $\beta \geqslant 0$ для $\mathcal{C}$ :

$$
\begin{gathered}
\rho=\rho_{0} \otimes\left(\bigotimes_{k=1}^{N} \rho_{k}\right), \\
\rho_{0}=\frac{e^{-\beta_{0} a^{*} a}}{Z\left(\beta_{0}\right)}, \quad \rho_{k}=\frac{e^{-\beta a^{*} a}}{Z(\beta)}, \quad k=1,2 \ldots, N .
\end{gathered}
$$

Этот случай соответствует матрице плотности $\rho_{L}$ в $(3.6)$ с диагональной матрицей $L=\operatorname{diag}\left(\beta_{0}, \beta, \ldots, \beta\right)$ и матрице плотности $\rho(\beta, \delta ; \xi)$ в представлении $(3.12)$, где $(\beta, \delta ; \xi)=\left(\beta, \beta_{0}-\beta ; e\right)$, т. е.

$$
\rho=\frac{\rho\left(\beta, \beta_{0}-\beta ; e\right)}{Z\left(\beta, \beta_{0}-\beta\right)}=\frac{1}{Z\left(\beta, \beta_{0}-\beta\right)} \exp \left[-\beta_{0} b_{0}^{*} b_{0}-\beta \sum_{j=1}^{N} b_{j}^{*} b_{j}\right] .
$$

Здесь $e^{\text {tr }}=(1,0, \ldots, 0) \in \mathbb{C}^{N+1}$ и

$$
Z\left(\beta, \beta_{0}-\beta\right)=Z\left(\beta_{0}\right) Z(\beta)^{N}=\frac{1}{\left(1-e^{-\beta_{0}}\right)\left(1-e^{-\beta}\right)^{N}} .
$$

Непосредственное применение к состоянию (3.20) (или (3.21)) формул (3.14), (3.15) и леммы 3.1 при $\xi=e$ (т. е. при $\left.\langle\xi, \xi\rangle=1,\langle\xi, \zeta\rangle=\zeta_{0}\right)$ приводит к следующему утверждению.

ЛЕмма 3.3. Характеристическая функция состояния (3.20) (или (3.21)) имеет вид

$$
\begin{aligned}
\omega_{\rho}(W(\zeta)) & =\operatorname{Tr}_{\mathscr{H}}[\rho W(\zeta)]= \\
& =\exp \left[-\frac{\left|\zeta_{0}\right|^{2}}{4}\left(\frac{1+e^{-\beta_{0}}}{1-e^{-\beta_{0}}}-\frac{1+e^{-\beta}}{1-e^{-\beta}}\right)-\frac{\langle\zeta, \zeta\rangle}{4} \frac{1+e^{-\beta}}{1-e^{-\beta}}\right],
\end{aligned}
$$

а энтропия равна

$$
S(\rho)=N s(\beta)+s\left(\beta_{0}\right)
$$


Поскольку согласно (2.12) матрица плотности $\rho(t)$ полной системы $\mathcal{S}+\mathcal{C}$ при $t=N \tau$ равна

$$
\rho(N \tau)=e^{-i \tau H_{N}} \ldots e^{-i \tau H_{1}} \rho e^{i \tau H_{1}} \ldots e^{i \tau H_{N}},
$$

для эволюции характеристической функции и энтропии получаем следующее утверждение.

Лемма 3.4. Характеристическая функция (2.15) состояния с матрищей плотности (3.24) равна

$$
\omega_{\rho(N \tau)}(W(\zeta))=\exp \left[-\frac{\left|\left(U_{1} \ldots U_{N} \zeta\right)_{0}\right|^{2}}{4}\left(\frac{1+e^{-\beta_{0}}}{1-e^{-\beta_{0}}}-\frac{1+e^{-\beta}}{1-e^{-\beta}}\right)-\frac{\langle\zeta, \zeta\rangle}{4} \frac{1+e^{-\beta}}{1-e^{-\beta}}\right]
$$

в то время как полная энтропия остается инвариантной:

$$
S(\rho(N \tau))=S(\rho)=N s(\beta)+s\left(\beta_{0}\right) .
$$

Здесь отображение $U_{1} \ldots U_{N}: \mathbb{C}^{N+1} \rightarrow \mathbb{C}^{N+1}$ дается формулами (2.19) и (2.20).

ДокАзАтЕльство. Из формулы (2.18) получаем, что

$$
\omega_{\rho(N \tau)}(W(\zeta))=\omega_{\rho}\left(W\left(U_{1} \ldots U_{N} \zeta\right)\right)
$$

Поскольку отображения $U_{j}: \mathbb{C}^{N+1} \rightarrow \mathbb{C}^{N+1}, j=1, \ldots, N$, являются унитарными (см. лемму 2.1), равенство (3.22) влечет (3.25). Наконец, получаем, что отображение (3.24) оставляет полную энтропию (3.23) инвариантной (см. выражение (3.4)). Лемма доказана.

Пусть $\omega$ и $\omega_{0}$ - два нормальных состояния на алгебре Вейля $\mathrm{KKC} \mathscr{A}(\mathscr{H})$ с матрицами плотности $\varrho$ и $\varrho_{0}$. Следуя работе [9] (см. также [10]), введем относительную энтропию состояния $\omega$ по отношению к состоянию $\omega_{0}$ как

$$
\operatorname{Ent}\left(\varrho \mid \varrho_{0}\right):=\operatorname{Tr}_{\mathscr{H}}\left[\varrho\left(\ln \varrho-\ln \varrho_{0}\right)\right] \geqslant 0
$$

ЛЕмма 3.5. Относительная энтропия состояния $\omega_{\rho(N \tau)}$ по отношению $\kappa$ состоянию $\omega_{\rho}$ равна

$$
\operatorname{Ent}(\rho(N \tau) \mid \rho)=\frac{\left(\beta_{0}-\beta\right)\left(e^{\beta_{0}}-e^{\beta}\right)}{\left(e^{\beta_{0}}-1\right)\left(e^{\beta}-1\right)}\left(1-|z|^{2 N}\right),
$$

где величина $z:=z(\tau)$ определяется выражениями (1.13) и (1.22).

ДокАЗАТЕЛЬСтво. Свойство цикличности следа дает

$$
\begin{aligned}
& \operatorname{Ent}(\rho(N \tau) \mid \rho)=\operatorname{Tr}_{\mathscr{H}}[\rho(N \tau)(\ln \rho(N \tau)-\ln \rho)]= \\
&= \operatorname{Tr}_{\mathscr{H}}\left[\rho\left(\ln \rho-e^{i \tau H_{1}} \ldots e^{i \tau H_{N}} \ln \rho e^{-i \tau H_{N}} \ldots e^{-i \tau H_{1}}\right)\right]= \\
&= \frac{\beta-\beta_{0}}{Z\left(\beta, \beta_{0}-\beta\right)} \times \\
& \quad \times \operatorname{Tr}_{\mathscr{H}}\left[e^{-\beta_{0} b_{0}^{*} b_{0}-\beta \sum_{j=1}^{N} b_{j}^{*} b_{j}}\left(b_{0}^{*} b_{0}-e^{i \tau H_{1}} \ldots e^{i \tau H_{N}} b_{0}^{*} b_{0} e^{-i \tau H_{N}} \ldots e^{-i \tau H_{1}}\right)\right] .
\end{aligned}
$$


Заметим, что $b_{0}^{*} b_{0}=\langle b, e\rangle\langle e, b\rangle$ согласно (2.4). Поэтому из (2.23) следует, что

$$
\begin{aligned}
& e^{i \tau H_{1}} \ldots e^{i \tau H_{N}} b_{0}^{*} b_{0} e^{-i \tau H_{N}} \ldots e^{-i \tau H_{1}}= \\
& =\sum_{k=0}^{N}\left(U_{1} \ldots U_{N} e\right)_{k} b_{k}^{*} \sum_{k^{\prime}=0}^{N} \overline{\left(U_{1} \ldots U_{N} e\right)_{k^{\prime}} b_{k^{\prime}}}
\end{aligned}
$$

Заметим также, что из калибровочной инвариантности состояния $\rho$ следует правило отбора

$$
\frac{1}{Z\left(\beta, \beta_{0}-\beta\right)} \operatorname{Tr}_{\mathscr{H}}\left[e^{-\beta_{0} b_{0}^{*} b_{0}-\beta \sum_{j=1}^{N} b_{j}^{*} b_{j}} b_{k}^{*} b_{k^{\prime}}\right]=0 \quad \text { при } \quad k \neq k^{\prime} .
$$

Согласно этому правилу после подстановки (3.29) в (3.28) в математическом ожидании выживают только диагональные члены, в которых $k=k^{\prime}$ :

$$
\begin{aligned}
& \operatorname{Ent}(\rho(N \tau) \mid \rho)= \\
& \qquad=\frac{\beta-\beta_{0}}{Z\left(\beta, \beta_{0}-\beta\right)} \operatorname{Tr}_{\mathscr{H}}\left[e^{-\beta_{0} b_{0}^{*} b_{0}-\beta \sum_{j=1}^{N} b_{j}^{*} b_{j}}\left(b_{0}^{*} b_{0}-\sum_{k=0}^{N}\left|\left(U_{1} \ldots U_{N} e\right)_{k}\right|^{2} b_{k}^{*} b_{k}\right)\right] .
\end{aligned}
$$

Наконец, с учетом леммы 2.1, выражений (2.19), (2.20), а также (3.4) получаем

$$
\begin{aligned}
& \operatorname{Ent}(\rho(N \tau) \mid \rho)=\frac{\beta-\beta_{0}}{Z\left(\beta, \beta_{0}-\beta\right)} \times \\
& \times \operatorname{Tr}_{\mathscr{H}}\left[e^{-\beta_{0} b_{0}^{*} b_{0}-\beta \sum_{j=1}^{N} b_{j}^{*} b_{j}}\left(\left(1-|z|^{2 N}\right) b_{0}^{*} b_{0}-\sum_{k=1}^{N}|w|^{2}|z|^{2 N-2 k} b_{k}^{*} b_{k}\right)\right]= \\
& \quad=\frac{\left(\beta_{0}-\beta\right)\left(e^{\beta_{0}}-e^{\beta}\right)}{\left(e^{\beta_{0}}-1\right)\left(e^{\beta}-1\right)}\left(1-|z|^{2 N}\right),
\end{aligned}
$$

что доказывает (3.27).

ЗАмЕчАниЕ 3.1. Относительная энтропия, которая определяется соотношением (3.26), неотрицательна. В отличие от инвариантной полной энтропии (см. лемму 3.4) отосительная энтропия (3.27) монотонно возрастает со временем $(N \tau \rightarrow \infty)$ при $|z|<1$ (см. лемму 1.1 и замечание 1.1 ). При этом

$$
\lim _{N \rightarrow \infty} \operatorname{Ent}(\rho(N \tau) \mid \rho)=\left(\beta-\beta_{0}\right)\left(\frac{1}{e^{\beta_{0}}-1}-\frac{1}{e^{\beta}-1}\right) \geqslant 0
$$

и предел положителен при $\beta_{0} \neq \beta$. Предел (3.31) дает асимптотическое значение для производства энтропии, если стартовать с состояния, соответствующего (3.20), а затем время устремить к бесконечности, $t=N \tau \rightarrow \infty$. 


\section{4. ДИНАМИКА ПОДСИСТЕМ}

\section{1. Сходимость к равновесному состоянию и локальная энтропия.}

Подсистема $\mathcal{S}$. Начнем с простейшей подсистемы $\mathcal{S}$. Пусть начальное состояние полной системы $\mathcal{S}+\mathcal{C}$ в $(1.1)$ представляет собой тензорное произведение соответствующих матриц плотности, $\rho=\rho_{S} \otimes \rho_{C}$ (см. гипотезу 1 ). Тогда при $t \geqslant 0$ состояние $\omega_{\mathcal{S}}^{t}(\cdot)$ подсистемы $\mathcal{S}$ задается на $C^{*}$-алгебре Вейля $\mathscr{A}\left(\mathscr{H}_{0}\right)$ как

$$
\omega_{\mathcal{S}}^{t}(\cdot):=\omega_{\rho(t)}(\cdot \otimes \mathbb{1})
$$

Для $\zeta=(\alpha, 0, \ldots, 0) \in \mathbb{C}^{N+1}$ рассмотрим оператор Вейля $W(\zeta)=\hat{w}(\alpha) \otimes \mathbb{1} \otimes \ldots \otimes \mathbb{1}$, заданный в (2.3). В силу соотношения (2.8), теоремы 2.1 и выражения (4.1) для $t=m \tau($ при $1 \leqslant m \leqslant N)$ получаем

$$
\omega_{\mathcal{S}}^{m \tau}(\hat{w}(\alpha))=\omega_{\rho(m \tau)}(W(\zeta))=\omega_{\rho}\left(W\left(U_{1} \ldots U_{m} \zeta\right)\right) .
$$

Тогда для компонент $\left\{\left(U_{1} \ldots U_{m} \zeta\right)_{k}\right\}_{k=0}^{N}$ вектора $U_{1} \ldots U_{m} \zeta$ в (4.2) получаем

$$
\left(U_{1} \ldots U_{m} \zeta\right)_{k}= \begin{cases}e^{i m \tau \epsilon}(g z)^{m} \alpha, & k=0, \\ e^{i m \tau \epsilon} g w(g z)^{m-k} \alpha, & 1 \leqslant k<m, \\ e^{i m \tau \epsilon} g w \alpha, & k=m, \\ 0, & m<k \leqslant N,\end{cases}
$$

что следует из замечания 2.3 .

Если начальные матрицы плотности $\rho=\rho_{S} \otimes \rho_{C}$ соответствуют произведению квазисвободных гиббсовских состояний при различных температурах, как в (3.20), то из формулы (4.2) и леммы 3.3 следует, что

$$
\omega_{\mathcal{S}}^{m \tau}(\hat{w}(\alpha))=\exp \left[-\frac{|\alpha|^{2}}{4} \frac{1+e^{-\beta}}{1-e^{-\beta}}-\frac{\left|z^{m} \alpha\right|^{2}}{4}\left(\frac{1+e^{-\beta_{0}}}{1-e^{-\beta_{0}}}-\frac{1+e^{-\beta}}{1-e^{-\beta}}\right)\right] .
$$

Заметим, что для любого момента $t=m \tau$ состояние $\omega_{\mathcal{S}}^{m \tau}(\cdot)$ является квазисвободным гиббсовским равновесным состоянием с параметром $\beta^{*}(m \tau)$, удовлетворяющим уравнению

$$
\frac{1+e^{-\beta^{*}(m \tau)}}{1-e^{-\beta^{*}(m \tau)}}=|z|^{2 m} \frac{1+e^{-\beta_{0}}}{1-e^{-\beta_{0}}}+\left(1-|z|^{2 m}\right) \frac{1+e^{-\beta}}{1-e^{-\beta}} .
$$

Из этого уравнения вытекает либо неравенство $\beta \leqslant \beta^{*}(m \tau) \leqslant \beta_{0}$, либо неравенство $\beta_{0} \leqslant \beta^{*}(m \tau) \leqslant \beta$.

При $m \rightarrow \infty(N \rightarrow \infty)$ характеристическая функция Вейля (4.4) имеет предел:

$$
\lim _{m \rightarrow \infty} \omega_{\mathcal{S}}^{m \tau}(\hat{w}(\alpha))=\exp \left[-\frac{|\alpha|^{2}}{4} \frac{1+e^{-\beta}}{1-e^{-\beta}}\right] .
$$

Поэтому в пределе $t \rightarrow \infty$ подсистема $\mathcal{S}$ в процессе эволюции переходит из гиббсовского равновесного состояния с параметром $\beta_{0}$ к другому равновесному состоянию с параметром $\beta$, фиксированным цепочкой $\mathcal{C}$. 
Подсистема $\mathcal{S}_{1}$. Начальное состояние $\omega_{\mathcal{S}_{1}}^{0}(\cdot)=\left.\omega_{\mathcal{S}_{1}}^{t}(\cdot)\right|_{t=0}$ этой подсистемы соответствует одноточечной редуцированной матрице плотности или частичному следу на алгебре Вейля $\mathrm{KKC} \mathscr{A}\left(\mathscr{H}_{1}\right)$ :

$$
\omega_{\mathcal{S}_{1}}^{0}(\hat{w}(\alpha))=\omega_{\rho}\left(\mathbb{1} \otimes \hat{w}(\alpha) \otimes\left(\bigotimes_{k=2}^{N} \mathbb{1}\right)\right)=\exp \left[-\frac{|\alpha|^{2}}{4} \frac{1+e^{-\beta}}{1-e^{-\beta}}\right] .
$$

Теперь выберем вектор $\left(\zeta^{1}\right)^{\text {tr }}:=(0, \alpha, 0, \ldots, 0) \in \mathbb{C}^{N+1}$. Тогда

$$
\omega_{\mathcal{S}_{1}}^{m \tau}(\hat{w}(\alpha))=\omega_{\rho(m \tau)}\left(W\left(\zeta^{(1)}\right)\right)=\omega_{\rho_{S} \otimes \rho_{C}}\left(W\left(U_{1} \ldots U_{m} \zeta^{(1)}\right)\right)
$$

для $1<m \leqslant N$. Согласно замечанию 2.3 компоненты $\left\{\left(U_{1} \ldots U_{m} \zeta^{(1)}\right)_{k}\right\}_{k=0}^{N}$ равны

$$
\left(U_{1} \ldots U_{m} \zeta\right)_{k}= \begin{cases}e^{i m \tau \epsilon} g w \alpha, & k=0 \\ e^{i m \tau \epsilon} \delta_{k, 1} g \bar{z} \alpha, & 1 \leqslant k<m \\ 0, & m \leqslant k \leqslant N\end{cases}
$$

Тогда получаем

$$
\omega_{\mathcal{S}_{1}}^{m \tau}(\hat{w}(\alpha))=\exp \left[-\frac{|\alpha|^{2}}{4} \frac{1+e^{-\beta}}{1-e^{-\beta}}-\frac{|w \alpha|^{2}}{4}\left(\frac{1+e^{-\beta_{0}}}{1-e^{-\beta_{0}}}-\frac{1+e^{-\beta}}{1-e^{-\beta}}\right)\right]
$$

для любого $1<m \leqslant N$. Поэтому начальное состояние (4.7) переходит в состояние (4.10) после первого акта взаимодействия на интервале $[0, \tau)$, а дальнейшей эволюции данного состояния при $t>\tau$ не происходит.

Выражение (4.10) представляет собой характеристическую функцию квазисвободного гиббсовского равновесного состояния с параметром $\beta^{*}$, который удовлетворяет уравнению

$$
\frac{1+e^{-\beta^{*}}}{1-e^{-\beta^{*}}}=|w|^{2} \frac{1+e^{-\beta_{0}}}{1-e^{-\beta_{0}}}+\left(1-|w|^{2}\right) \frac{1+e^{-\beta}}{1-e^{-\beta}} .
$$

Из этого уравнения снова следует, что либо $\beta \leqslant \beta^{*} \leqslant \beta_{0}$, либо $\beta_{0} \leqslant \beta^{*} \leqslant \beta$.

Заметим, что эволюция подсистемы $\mathcal{S}_{1}$ имеет ясную интерпретацию: после одного акта взаимодействия в течение времени $t \in[0, \tau)$ подсистема $\mathcal{S}_{1}$ релаксирует к состоянию промежуточного равновесия с подсистемой $\mathcal{S}$. В результате происходит сдвиг начального параметра $\beta$ к $\beta^{*}$. Затем этот параметр не меняется со временем, поскольку при $t>\tau$ нет возмущений подсистемы $\mathcal{S}_{1}$.

Подсистема $\mathcal{S}_{m}$. При $1<m \leqslant N$ начальное состояние $\omega_{\mathcal{S}_{m}}^{0}(\cdot)=\left.\omega_{\mathcal{S}_{m}}^{t}(\cdot)\right|_{t=0}$ данной подсистемы определяется на алгебре Вейля $\mathrm{KKC} \mathscr{A}\left(\mathscr{H}_{m}\right)$ с помощью частичного следа:

$$
\omega_{\mathcal{S}_{m}}^{0}(\hat{w}(\alpha))=\omega_{\rho}\left(\left(\bigotimes_{k=0}^{m-1} \mathbb{1}\right) \otimes \hat{w}(\alpha) \otimes\left(\bigotimes_{k=m+1}^{N} \mathbb{1}\right)\right)=\exp \left[-\frac{|\alpha|^{2}}{4} \frac{1+e^{-\beta}}{1-e^{-\beta}}\right]
$$

Выберем теперь вектор $\left(\zeta^{m}\right)^{\operatorname{tr}}:=(0, \ldots, 0, \alpha, 0, \ldots, 0) \in \mathbb{C}^{N+1}$, где $\alpha$ стоит на $(m+1)$-м месте. Тогда теорема 2.1 дает

$$
\omega_{\mathcal{S}_{m}}^{m \tau}(\hat{w}(\alpha))=\omega_{\rho(m \tau)}\left(W\left(\zeta^{(m)}\right)\right)=\omega_{\rho_{S} \otimes \rho_{C}}\left(W\left(U_{1} \ldots U_{m} \zeta^{(m)}\right)\right) .
$$


Компоненты $\left\{\left(U_{1} \ldots U_{m} \zeta^{(m)}\right)_{k}\right\}_{k=0}^{N}$ равны

$$
\left(U_{1} \ldots U_{m} \zeta^{(m)}\right)_{k}= \begin{cases}e^{i m \tau \epsilon} g w(g z)^{m-1} \alpha, & k=0, \\ e^{i m \tau \epsilon} g^{2} w^{2}(g z)^{m-k-1} \alpha, & 1 \leqslant k<m, \\ e^{i m \tau \epsilon} g \bar{z} \alpha, & k=m, \\ 0, & m<k \leqslant N,\end{cases}
$$

что снова следует из замечания 2.3. Тогда эволюция состояния подсистемы $\mathcal{S}_{m}$ задается как

$$
\omega_{\mathcal{S}_{m}}^{m \tau}(\hat{w}(\alpha))=\exp \left[-\frac{|\alpha|^{2}}{4} \frac{1+e^{-\beta}}{1-e^{-\beta}}-\frac{|w \alpha|^{2}}{4}|z|^{2(m-1)}\left(\frac{1+e^{-\beta_{0}}}{1-e^{-\beta_{0}}}-\frac{1+e^{-\beta}}{1-e^{-\beta}}\right)\right] .
$$

Заметим, что взаимодействие при $t \in[(m-1) \tau, m \tau)$ выталкивает подсистему $\mathcal{S}_{m}$ из гиббсовского равновесного состояния (4.11), однако этот эффект идет на убыль при больших $m$ :

$$
\lim _{m \rightarrow \infty} \omega_{\mathcal{S}_{m}}^{m \tau}(\hat{w}(\alpha))=\exp \left[-\frac{|\alpha|^{2}}{4} \frac{1+e^{-\beta}}{1-e^{-\beta}}\right] .
$$

Как и выше, мы имеем здесь эволюцию квазисвободного гиббсовского равновесного состояния с зависящим от времени параметром обратной температуры $\beta^{* *}(m \tau)$, который удовлетворяет уравнению

$$
\frac{1+e^{-\beta^{* *}(m \tau)}}{1-e^{-\beta^{* *}(m \tau)}}=|w|^{2}|z|^{2(m-1)} \frac{1+e^{-\beta_{0}}}{1-e^{-\beta_{0}}}+\left(1-|w|^{2}|z|^{2(m-1)}\right) \frac{1+e^{-\beta}}{1-e^{-\beta}} .
$$

Как и выше, значение параметра $\beta^{* *}(m \tau)$ всегда лежит между $\beta_{0}$ и $\beta$.

Чтобы проанализировать эволюцию системы $\mathcal{S}_{m}$ и совпадение пределов (4.15) и (4.6), заметим, что состояние подсистемы $\mathcal{S}$ релаксирует к начальному состоянию цепочки $\mathcal{C}$ (см. соотношение (4.6)). Таким образом, после того как подсистема $\mathcal{S}_{m}$ провзаимодействует, т. е. в момент $t=m \tau$, значение параметра $\beta^{* *}(m \tau)$ лежит между $\beta$ и $\beta^{*}((m-1) \tau)$, поскольку из (4.5) и (4.16) следует, что

$$
\frac{1+e^{-\beta^{* *}(m \tau)}}{1-e^{-\beta^{* *}(m \tau)}}=|w|^{2} \frac{1+e^{-\beta^{*}((m-1) \tau)}}{1-e^{-\beta^{*}((m-1) \tau)}}+\left(1-|w|^{2}\right) \frac{1+e^{-\beta}}{1-e^{-\beta}} .
$$

Как и в случае $m=1$, дальнейшей эволюции не происходит: $\omega_{\mathcal{S}_{m}}^{n \tau}=\omega_{\mathcal{S}_{m}}^{m \tau}$ при $n \geqslant m$.

Теперь рассмотрим составные подсистемы $\mathcal{S}+\mathcal{S}_{m}$ и $\mathcal{S}_{m-n}+\mathcal{S}_{m}$. Нашей целью является изучение окончательных корреляций, возникающих из-за повторяющегося взаимодействия с подсистемой $\mathcal{S}$.

Подсистема $\mathcal{S}+\mathcal{S}_{m}$. Начальное состояние $\omega_{\mathcal{S}+\mathcal{S}_{m}}^{0}(\cdot)=\left.\omega_{\mathcal{S}+\mathcal{S}_{m}}^{t}(\cdot)\right|_{t=0}$ такой составной подсистемы при $1<m \leqslant N$ определяется частичным следом на $C^{*}$-алгебре Вейля $\mathscr{A}\left(\mathscr{H}_{0} \otimes \mathscr{H}_{m}\right) \approx \mathscr{A}\left(\mathscr{H}_{0}\right) \otimes \mathscr{A}\left(\mathscr{H}_{m}\right)$ следующим образом:

$$
\begin{aligned}
\omega_{\mathcal{S}+\mathcal{S}_{m}}^{0}\left(\hat{w}\left(\alpha_{0}\right) \otimes \hat{w}\left(\alpha_{1}\right)\right) & :=\omega_{\rho}\left(\hat{w}\left(\alpha_{0}\right) \otimes\left(\bigotimes_{k=1}^{m-1} \mathbb{1}\right) \otimes \hat{w}\left(\alpha_{1}\right) \otimes\left(\bigotimes_{k=m+1}^{N} \mathbb{1}\right)\right)= \\
& =\exp \left[-\frac{\left|\alpha_{0}\right|^{2}}{4} \frac{1+e^{-\beta_{0}}}{1-e^{-\beta_{0}}}\right] \exp \left[-\frac{\left|\alpha_{1}\right|^{2}}{4} \frac{1+e^{-\beta}}{1-e^{-\beta}}\right] .
\end{aligned}
$$


Это выражение представляет собой характеристическую функцию состояния-произведения, соответствующего двум изолированным системам с различными температурами. Пусть $\left(\zeta^{(0, m)}\right)^{\operatorname{tr}}:=\left(\alpha_{0}, 0, \ldots, 0, \alpha_{1}, 0, \ldots, 0\right) \in \mathbb{C}^{N+1}$, где $\alpha_{1}$ стоит на $(m+1)$-м месте. Тогда согласно теореме 2.1 имеем

$$
\omega_{\mathcal{S}+\mathcal{S}_{m}}^{m \tau}\left(\hat{w}\left(\alpha_{0}\right) \otimes \hat{w}\left(\alpha_{1}\right)\right)=\omega_{\rho(m \tau)}\left(W\left(\zeta^{(0, m)}\right)\right)=\omega_{\rho_{S} \otimes \rho_{C}}\left(W\left(U_{1} \ldots U_{m} \zeta^{(0, m)}\right)\right) .
$$

Компоненты $\left\{\left(U_{1} \ldots U_{m} \zeta^{(0, m)}\right)_{k}\right\}_{k=0}^{N}$ выводятся на основании замечания 2.3:

$$
\left(U_{1} \ldots U_{m} \zeta^{(0, m)}\right)_{k}= \begin{cases}e^{i m \tau \epsilon}(g z)^{m-1}\left[g z \alpha_{0}+g w \alpha_{1}\right], & k=0, \\ e^{i m \tau \epsilon}(g z)^{m-k-1} g^{2}\left[w z \alpha_{0}+w^{2} \alpha_{1}\right], & 1 \leqslant k<m, \\ e^{i m \tau \epsilon}\left[g w \alpha_{0}+g \bar{z} \alpha_{1}\right], & k=m, \\ 0, & m<k \leqslant N .\end{cases}
$$

С учетом (2.8) при $m \rightarrow \infty$ получаем

$$
\begin{aligned}
\omega_{\mathcal{S}+\mathcal{S}_{m}}^{m \tau}\left(\hat{w}\left(\alpha_{0}\right) \otimes \hat{w}\left(\alpha_{1}\right)\right)= \\
=\exp \left[-\frac{\left|z \alpha_{0}+w \alpha_{1}\right|^{2}}{4}|z|^{2(m-1)} \frac{1+e^{-\beta_{0}}}{1-e^{-\beta_{0}}}\right] \times \\
\quad \times \exp \left[-\frac{\left|z \alpha_{0}+w \alpha_{1}\right|^{2}}{4}\left(1-|z|^{2(m-1)}\right) \frac{1+e^{-\beta}}{1-e^{-\beta}}\right] \exp \left[-\frac{\left|w \alpha_{0}+\bar{z} \alpha_{1}\right|^{2}}{4} \frac{1+e^{-\beta}}{1-e^{-\beta}}\right] \rightarrow \\
\rightarrow \exp \left[-\frac{\left|\alpha_{0}\right|^{2}+\left|\alpha_{1}\right|^{2}}{4} \frac{1+e^{-\beta}}{1-e^{-\beta}}\right] .
\end{aligned}
$$

Таким образом, в данном пределе составная подсистема $\mathcal{S}+\mathcal{S}_{m}$ в процессе эволюции переходит от произведения двух квазисвободных равновесных состояний (4.18) с различными параметрами $\beta_{0}$ и $\beta$ к произведению квазисвободных равновесных состояний с тем же параметром $\beta$, которые определяются повторяющимся взаимодействием с цепочкой $\mathcal{C}$ при $m \rightarrow \infty$. Интерпретация аналогична случаю подсистемы $\mathcal{S}_{m}$.

Подсистема $\mathcal{S}_{m-n}+\mathcal{S}_{m}$. Пусть $1<m-n<m \leqslant N$. Тогда начальное состояние $\left.\omega_{\mathcal{S}_{m-n}+\mathcal{S}_{m}}^{t}(\cdot)\right|_{t=0}$ этой составной подсистемы представляет собой частичный след на $C^{*}$-алгебре Вейля $\mathscr{A}\left(\mathscr{H}_{m-n} \otimes \mathscr{H}_{m}\right) \approx \mathscr{A}\left(\mathscr{H}_{m-n}\right) \otimes \mathscr{A}\left(\mathscr{H}_{m}\right):$

$$
\begin{aligned}
& \omega_{\mathcal{S}_{m-n}+\mathcal{S}_{m}}^{0}\left(\hat{w}\left(\alpha_{1}\right) \otimes \hat{w}\left(\alpha_{2}\right)\right):= \\
& \quad:=\omega_{\rho}\left(\left(\bigotimes_{k=0}^{m-n-1} \mathbb{1}\right) \otimes \hat{w}\left(\alpha_{1}\right) \otimes\left(\bigotimes_{k=m-n+1}^{m-1} \mathbb{1}\right) \otimes \hat{w}\left(\alpha_{2}\right) \otimes\left(\bigotimes_{k=m+1}^{N} \mathbb{1}\right)\right)= \\
& \quad=\exp \left[-\frac{\left|\alpha_{1}\right|^{2}}{4} \frac{1+e^{-\beta}}{1-e^{-\beta}}\right] \exp \left[-\frac{\left|\alpha_{2}\right|^{2}}{4} \frac{1+e^{-\beta}}{1-e^{-\beta}}\right] .
\end{aligned}
$$

Это характеристическая функция состояния-произведения, соответствующего двум изолированным системам с одинаковыми температурами.

Зададим вектор $\left(\zeta^{(m-n, m)}\right)^{\operatorname{tr}}:=\left(0,0, \ldots, 0, \alpha_{1}, 0, \ldots, 0, \alpha_{2}, 0, \ldots, 0\right) \in \mathbb{C}^{N+1}$, где $\alpha_{1}$ стоит на $(m-n+1)$-м месте, а $\alpha_{2}$ - на $(m+1)$-м месте. Тогда

$$
\begin{aligned}
\omega_{\mathcal{S}_{m-n} \tau}^{m \tau}+\mathcal{S}_{m}\left(\hat{w}\left(\alpha_{1}\right) \otimes \hat{w}\left(\alpha_{2}\right)\right) & =\omega_{\rho(m \tau)}\left(W\left(\zeta^{(m-n, m)}\right)\right)= \\
& =\omega_{\rho_{S} \otimes \rho_{C}}\left(W\left(U_{1} \ldots U_{m} \zeta^{(m-n, m)}\right)\right) .
\end{aligned}
$$


Компоненты $\left\{\left(U_{1} \ldots U_{m} \zeta^{(m-n, m)}\right)_{k}\right\}_{k=0}^{N}$ можно вычислить на основании замечания 2.3:

$$
\begin{aligned}
& \left(U_{1} \ldots U_{m} \zeta^{(m-n, m)}\right)_{k}= \\
& \quad= \begin{cases}e^{i m \tau \epsilon}(g z)^{m-n-1} g w\left[\alpha_{1}+(g z)^{n} \alpha_{2}\right], & k=0, \\
e^{i m \tau \epsilon}\left[g^{2} w^{2}(g z)^{m-n-k-1} \alpha_{1}+g^{2} w^{2}(g z)^{m-k-1} \alpha_{2}\right], & 1 \leqslant k<m-n, \\
e^{i m \tau \epsilon}\left[g \bar{z} \alpha_{1}+g^{2} w^{2}(g z)^{m-k-1} \alpha_{2}\right], & k=m-n, \\
e^{i m \tau \epsilon} g^{2} w^{2}(g z)^{m-k-1} \alpha_{2}, & m-n<k<m, \\
e^{i m \tau \epsilon} g \bar{z} \alpha_{2}, & k=m, \\
0, & m<k \leqslant N .\end{cases}
\end{aligned}
$$

Если $m \rightarrow \infty$, то при любом фиксированном $n$ для (4.23) получаем

$$
\begin{aligned}
\omega_{\mathcal{S}_{m-n}+\mathcal{S}_{m}}^{m \tau} & \left(\hat{w}\left(\alpha_{1}\right) \otimes \hat{w}\left(\alpha_{2}\right)\right)= \\
= & \exp \left[-\frac{\left|\alpha_{1}+(g z)^{n+1} \alpha_{2}\right|^{2}}{4}|w|^{2}|z|^{2(m-n-1)} \frac{1+e^{-\beta_{0}}}{1-e^{-\beta_{0}}}\right] \times \\
& \times \exp \left[-\frac{\left|\alpha_{1}\right|^{2}}{4}\left[|w|^{2}\left(1-|z|^{2(m-n-1)}\right)+|z|^{2}\right] \frac{1+e^{-\beta}}{1-e^{-\beta}}\right] \times \\
& \times \exp \left[-\frac{\left|\alpha_{2}\right|^{2}}{4}\left(1-|w|^{2}|z|^{2(m-1)}\right) \frac{1+e^{-\beta}}{1-e^{-\beta}}\right] \rightarrow \\
\rightarrow & \exp \left[-\frac{\left|\alpha_{1}\right|^{2}+\left|\alpha_{2}\right|^{2}}{4} \frac{1+e^{-\beta}}{1-e^{-\beta}}\right] .
\end{aligned}
$$

Следовательно, в этом пределе составная подсистема $\mathcal{S}_{m-n}+\mathcal{S}_{m}$ в процессе эволюции переходит из начального произведения двух квазисвободных равновесных состояний (4.22) к тому же финальному состоянию, хотя при конечных $m$ эволюция (4.24) является нетривиальной. Это также нетрудно понять, учитывая наш анализ подсистемы $\mathcal{S}_{m}$ и подсистемы $\mathcal{S}+\mathcal{S}_{m}$.

Теперь рассмотрим случай фиксированного $s:=m-n \geqslant 1$. Тогда предел в (4.24) рассчитывается как

$$
\begin{aligned}
\lim _{m \rightarrow \infty} \omega_{\mathcal{S}_{s}+\mathcal{S}_{m}}^{m \tau}\left(\hat{w}\left(\alpha_{1}\right) \otimes \hat{w}\left(\alpha_{2}\right)\right)= \\
=\exp \left[-\frac{\left|\alpha_{1}\right|^{2}}{4}|w|^{2}|z|^{2(s-1)}\left(\frac{1+e^{-\beta_{0}}}{1-e^{-\beta_{0}}}-\frac{1+e^{-\beta}}{1-e^{-\beta}}\right)\right] \times \\
\quad \times \exp \left[-\frac{\left|\alpha_{1}\right|^{2}}{4} \frac{1+e^{-\beta}}{1-e^{-\beta}}\right] \exp \left[-\frac{\left|\alpha_{2}\right|^{2}}{4} \frac{1+e^{-\beta}}{1-e^{-\beta}}\right]= \\
=\exp \left[-\frac{\left|\alpha_{1}\right|^{2}}{4} \frac{1+e^{-\beta^{* *}(s \tau)}}{1-e^{-\beta^{* *}(s \tau)}}\right] \exp \left[-\frac{\left|\alpha_{2}\right|^{2}}{4} \frac{1+e^{-\beta}}{1-e^{-\beta}}\right],
\end{aligned}
$$

где $\beta^{* *}(s \tau)$ удовлетворяет уравнению (4.16). Таким образом, в этом случае предельное состояние (4.25) представляет собой произведение квазисвободных гиббсовских состояний с различными параметрами $\beta^{* *}(s \tau)$ и $\beta$. Это означает, что подсистема $\mathcal{S}_{s}$ сохраняет память о возмущении в момент времени $t=s \tau$, когда параметр $\beta^{*}(s \tau)(4.5)$ подсистемы $\mathcal{S}$ еще отличался от $\beta$.

Заметим, что (4.25) совпадает с состоянием-произведением (4.22) при $s \rightarrow \infty$. 
Подсистема $\mathcal{S}_{\sim n}$. Чтобы определить $\mathcal{S}_{\sim n}$ при $0 \leqslant n \leqslant k \leqslant N$, разделим полную систему в момент времени $t=k \tau$ на две подсистемы $\mathcal{S}_{n, k}+\mathcal{C}_{n, k}$. Здесь

$$
\mathcal{S}_{n, k}:=\mathcal{S}+\mathcal{S}_{k}+\mathcal{S}_{k-1}+\cdots+\mathcal{S}_{k-n+1} \quad\left(\mathcal{S}_{0, k}:=\mathcal{S}\right)
$$

тогда как (см. определения в п. 1.1)

$$
\mathcal{C}_{n, k}:=\mathcal{S}_{N}+\cdots+\mathcal{S}_{k+1}+\mathcal{S}_{k-n}+\cdots+\mathcal{S}_{1}
$$

Будем считать, что $\mathcal{S}_{\sim n}$ является цельным "объектом", который в момент времени $t=k \tau(k=n, n+1, \ldots, N)$ совпадает с $\mathcal{S}_{n, k}$. По мере того как время пробегает различные значения, элементарные подсистемы $\mathcal{S}_{k}$ в $\mathcal{S}_{\sim n}$ меняются. Рассмотрим поведение системы $\mathcal{S}_{\sim n}$ при больших $t=k \tau$, т. е. зависимость "состояния" подсистемы $\mathcal{S}_{n, k}$ от $k$ при $t=k \tau$.

Для любого фиксированного $t=k \tau$ гильбертово пространство $\mathscr{H}$ можно разложить в тензорное произведение $\mathscr{H}=\mathscr{H}_{s} \otimes \mathscr{H}_{c}$. Здесь $\mathscr{H}_{s}-$ гильбертово пространство подсистемы (4.26), а $\mathscr{H}_{c}$ - гильбертово пространство подсистемы (4.27):

$$
\mathscr{H}_{s}:=\mathscr{H}_{0} \otimes\left(\bigotimes_{j=1}^{n} \mathscr{H}_{k-j+1}\right), \quad \mathscr{H}_{c}:=\left(\bigotimes_{j=1}^{k-n} \mathscr{H}_{j}\right) \otimes\left(\bigotimes_{j=k+1}^{N} \mathscr{H}_{j}\right) .
$$

Для матрицы плотности $\varrho$ на $\mathscr{H}$ введем редуцированную матрицу плотности $\varrho_{s}$ на пространстве $\mathscr{H}_{s}$ как частичный след по $\mathscr{H}_{c}$ :

$$
\varrho_{s}:=\operatorname{Tr}_{\mathscr{H}_{c}} \varrho .
$$

Чтобы избежать возможных недоразумений, связанных с тем, что все пространства $\mathscr{H}_{j}, j=0,1, \ldots$, тождественны фоковскому пространству $\mathscr{F}$, и с тем, что компоненты изменяются со временем, будем рассматривать алгебру Вейля на подсистеме и соответствующую редуцированную матрицу плотности $\rho \in \mathfrak{C}_{1}(\mathscr{H})$ следующим образом. При $n \leqslant N$ на пространстве Фока $\mathscr{F} \otimes(n+1)$ рассмотрим оператор Вейля

$$
W_{n}(\zeta)=\exp \left[i \frac{\langle\zeta, \tilde{b},\rangle+\langle\tilde{b}, \zeta\rangle}{\sqrt{2}}\right]
$$

где $\zeta \in \mathbb{C}^{n+1}, \tilde{b}_{0}, \ldots, \tilde{b}_{n}$ и $\tilde{b}_{0}^{*}, \ldots, \tilde{b}_{n}^{*}$ - операторы уничтожения и рождения на $\mathscr{F} \otimes(n+1)$, удовлетворяющие соответствующим $\mathrm{KKC}$, и

$$
\langle\zeta, \tilde{b}\rangle=\sum_{j=0}^{n} \bar{\zeta}_{j} \tilde{b}_{j}, \quad\langle\tilde{b}, \zeta\rangle=\sum_{j=0}^{n} \zeta_{j} \tilde{b}_{j}^{*}
$$

Через $\mathscr{A}\left(\mathscr{F}^{\otimes(n+1)}\right)$ обозначим $C^{*}$-алгебру, порожденную операторами Вейля (4.30). Для любого подмножества $J \subset\{1,2, \ldots, N\}$ определим операцию взятия частичного следа

$$
R^{J}: \mathfrak{C}_{1}\left(\mathscr{F}^{\otimes(N+1)}\right) \ni \rho \mapsto R^{J} \rho \in \mathfrak{C}_{1}\left(\mathscr{F}^{\otimes(N+1-|J|)}\right)
$$

посредством равенства

$$
\omega_{R^{J} \rho}\left(W_{N-|J|}(\zeta)\right)=\omega_{\rho}\left(W_{N}\left(r_{J} \zeta\right)\right)
$$


Здесь отображение

$$
r_{J}: \mathbb{C}^{N+1-|J|} \ni \zeta \mapsto r_{J} \zeta \in \mathbb{C}^{N+1}
$$

задается как

$$
\left(r_{J} \zeta\right)_{j}:= \begin{cases}\zeta_{0}, & j=0, \\ 0, & j \in J, \\ \zeta_{j-|\{i \in J \mid i<j\}|} & \text { в иных случаях, }\end{cases}
$$

где $|A|$ обозначает мощность множества $A$.

Поскольку все пространства $\mathscr{H}_{1}, \mathscr{H}_{2}, \ldots$ тождественны $\mathscr{F}$, мы можем не заботиться о том, чтобы различать $\bigotimes_{j \in\{0,1, \ldots, N\} \backslash J} \mathscr{H}_{j}$ и $\bigotimes_{j \in\{0,1, \ldots, N\} \backslash J^{\prime}} \mathscr{H}_{j}$, если $J \neq J^{\prime}$, но $|J|=\left|J^{\prime}\right|$, и рассматривать их как одно и то же пространство $\mathscr{F} \otimes(N+1-|J|)$. Наоборот, следует различать проекции

$$
\bigotimes_{j=0}^{N} \mathscr{H}_{j} \mapsto \bigotimes_{j \in\{0,1, \ldots, N\} \backslash J} \mathscr{H}_{j}
$$

для различных подмножеств $J \subset\{1,2, \ldots, N\}$ с одной и той же мощностью $|J|$.

Мы рассматриваем систему $\mathcal{S}_{n, k}$ в момент времени $t=k \tau(k=n, n+1, \ldots)$ как результат временной эволюции цельной подсистемы $\mathcal{S}_{\sim n}$, поэтому определим состояние системы $\mathcal{S}_{\sim n}$ в момент времени $t=k \tau$ с помощью редуцированной матрицы плотности $\left\{\rho_{s}(k \tau)\right\}_{k \geqslant n}$ данной подсистемы следующим образом (см. соотношение (2.12)):

$$
\rho_{s}(k \tau):=R^{\{1, \ldots, k-n, k+1, \ldots, N\}}(\rho(k \tau))=R^{\{1, \ldots, k-n, k+1, \ldots, N\}} T_{k \tau}(\rho) .
$$

С учетом леммы 3.4 и тождества $\left\langle r_{J} \zeta, r_{J} \zeta\right\rangle_{\mathbb{C}^{N+1}}=\langle\zeta, \zeta\rangle_{\mathbb{C}^{N+1-|J|}}$ немедленно получаем следующий результат.

ЛЕмма 4.1. Для начальной матрицы плотности (3.20) имеет место равенство

$$
\begin{aligned}
\omega_{\rho_{s}(k \tau)}\left(W_{n}(\zeta)\right) & =\omega_{R^{J_{n, k}} \rho(k \tau)}\left(W_{n}(\zeta)\right)= \\
& =\exp \left[-\frac{\left|\left(U_{1} \ldots U_{k} r_{J_{n, k}} \zeta\right)_{0}\right|^{2}}{4}\left(\frac{1+e^{-\beta_{0}}}{1-e^{-\beta_{0}}}-\frac{1+e^{-\beta}}{1-e^{-\beta}}\right)-\frac{\langle\zeta, \zeta\rangle}{4} \frac{1+e^{-\beta}}{1-e^{-\beta}}\right],
\end{aligned}
$$

где $J_{n, k}=\{1,2, \ldots, k-n, k+1, \ldots, N\}$.

Рассмотрим пределы $k \rightarrow \infty$ и $N \rightarrow \infty$ (для $k \leqslant N$ ) при фиксированном $n$. Для этого заметим, что из формулы $(2.19)$ следует, что $\left(U_{1} \ldots U_{k} r_{J_{n, k}} \zeta\right)_{0} \rightarrow 0$, кроме того, $|z|<1$. Из леммы 4.1 вытекает, что

$$
\lim _{k \rightarrow \infty} \omega_{\rho_{s}(k \tau)}\left(W_{n}(\zeta)\right)=\exp \left[-\frac{\langle\zeta, \zeta\rangle}{4} \frac{1+e^{-\beta}}{1-e^{-\beta}}\right]=\omega_{\rho_{n}^{(\beta)}}\left(W_{n}(\zeta)\right) .
$$

Здесь согласно теореме Араки-Сигала и с учетом неприводимости алгебры ККС $\mathscr{A}(\mathscr{F} \otimes(n+1))$ матрица плотности имеет вид

$$
\rho_{n}^{(\beta)}=\frac{1}{Z(\beta)^{n+1}} \exp \left[-\beta \sum_{j=0}^{n} \tilde{b}_{j}^{*} \tilde{b}_{j}\right] Z(\beta)=\left(1-e^{-\beta}\right)^{-1} .
$$

Таким образом, мы доказали следующее утверждение. 
Теорема 4.1. Пусть началъное состояние полной системы $\mathcal{S}+\mathcal{C}$ определяется матрицей плотности (3.21): $\rho=\rho\left(\beta, \beta_{0}-\beta ; е\right)$. Тогда для любого фиксированного $n$ состояние $\omega_{\rho_{s}(k \tau)}(\cdot)$ подсистемы $\mathcal{S}_{n, k}$ сходится $\kappa$ равновесному гиббсовскому состояния $\omega_{\rho_{n}^{(\beta)}}(\cdot)$ при $k \rightarrow \infty$ в слабой* топологии для состояний из $\mathscr{A}(\mathscr{F} \otimes(n+1))$.

Теорема 4.2. При условиях, аналогичных условиям теоремы 4.1,

$$
\lim _{k \rightarrow \infty} S\left(\rho_{s}(k \tau)\right)=S\left(\rho_{n}^{(\beta)}\right) .
$$

ДокАЗАтЕЛЬство. Зададим вектор $\xi_{n, k} \in \mathbb{C}^{n+1}$ как $\left(U_{1} \ldots U_{k} r_{J_{n, k}} \zeta\right)_{0}=:\left\langle\xi_{n, k}, \zeta\right\rangle$. Тогда $\left\langle\xi_{n, k}, \xi_{n, k}\right\rangle \rightarrow 0$ при $k \rightarrow \infty$ для фиксированного $n$. Отсюда, используя лемму 3.2 и лемму 4.1 , получаем

$$
\begin{aligned}
S\left(\rho_{s}(k \tau)\right) & =n \sigma\left(\frac{1+e^{-\beta}}{1-e^{-\beta}}\right)+\sigma\left(\frac{1+e^{-\beta}}{1-e^{-\beta}}+\left\langle\xi_{n, k}, \xi_{n, k}\right\rangle\left(\frac{1+e^{-\beta_{0}}}{1-e^{-\beta_{0}}}-\frac{1+e^{-\beta}}{1-e^{-\beta}}\right)\right) \rightarrow \\
& \rightarrow(n+1) \sigma\left(\frac{1+e^{-\beta}}{1-e^{-\beta}}\right)=S\left(\rho_{n}^{(\beta)}\right) .
\end{aligned}
$$

Теорема доказана.

ЗАМЕЧАНИЕ 4.1. Локальная энтропия (4.35) подсистемы $\mathcal{S}_{\sim n}(4.26)$ убывает или возрастает, если соответственно $\beta>\beta_{0}$ или $\beta<\beta_{0}$.

\section{2. Повторяющееся возмущение в пределе короткого времени взаимо-}

действия. Отметим, что результаты п. 4.1 получены в основном благодаря тому, что мы знаем явный вид начальной матрицы плотности $(3.20)$ полной системы $\mathcal{S}+\mathcal{C}$. Покажем, что отсутствие подобной информации не оказывает решающего влияния на некоторые результаты, касающиеся сходимости к равновесному состоянию при $t=N \tau \rightarrow \infty$, если рассматривать повторяющееся взаимодействие в пределе короткого времени взаимодействия: при $\tau \rightarrow 0$.

Рассмотрим данный предел для подсистемы $\mathcal{S}$. По-прежнему будем считать, что начальное состояние системы $\mathcal{S}+\mathcal{C}$ есть состояние-произведение с матрицей плотности (см. соотношение (2.7))

$$
\rho=\rho_{0} \otimes\left(\bigotimes_{k=1}^{N} \rho_{k}\right) \in \mathfrak{C}_{1}(\mathscr{H}),
$$

однако ослабим условия на $\rho_{0}$ и на $\left\{\rho_{k}\right\}_{k=1}^{N}$ следующим образом:

$$
\begin{gathered}
\rho_{1}=\rho_{2}=\cdots=\rho_{N} \in \mathfrak{C}_{1}(\mathscr{F}), \quad \rho_{0} \in \mathfrak{C}_{1}(\mathscr{F}), \\
\operatorname{Tr}_{\mathscr{F}}\left(\rho_{1} a\right)=\operatorname{Tr}_{\mathscr{F}}\left(\rho_{1} a^{2}\right)=\operatorname{Tr}_{\mathscr{F}}\left(\rho_{1} a^{*}\right)=\operatorname{Tr}_{\mathscr{F}}\left(\rho_{1} a^{* 2}\right)=0, \\
\operatorname{Tr}_{\mathscr{F}}\left[\rho_{1}\left(a^{*} a\right)^{2}\right]<\infty .
\end{gathered}
$$

ЗАмЕчаниЕ 4.2. Гипотезы (h1)-(h3) будут выполнены, если матрицы плотности $\left\{\rho_{k}\right\}_{k=0}^{N}$ соответствуют калибровочно-инвариантным квазисвободным состояниям с параметром $\beta_{0}$ при $k=0$ и параметром $\beta$ при $k=1,2, \ldots, N$ (см. соотношения (3.20)). Тогда гипотеза (h2) обусловлена калибровочной инвариантностью, а для гипотезы (h3) получаем

$$
\operatorname{Tr}_{\mathscr{F}}\left[\rho_{k}\left(a^{*} a\right)^{2}\right]=2 n_{\beta}^{2}+n_{\beta},
$$

где $n_{\beta}=\operatorname{Tr}_{\mathscr{F}}\left(\rho_{k} a^{*} a\right)=\left(e^{\beta}-1\right)^{-1}, k=1, \ldots, N$. 
Пусть $\xi a^{*}+\bar{\xi} a=U\left|\xi a^{*}+\bar{\xi} a\right|-$ полярное разложение замкнутого оператора $\xi a^{*}+\bar{\xi} a$. Здесь $\left|\xi a^{*}+\bar{\xi} a\right|-$ положительный самосопряженный оператор, а $U$ - частичная изометрия на пространстве $\mathscr{F}$. Тогда имеет место следующая лемма.

Лемма 4.2. При выполнении гипотез (h1)-(h3) для всех $k=1, \ldots, N$ мъ имеем следующие ограничения:

$$
\begin{gathered}
\operatorname{Tr}_{\mathscr{F}}\left(\rho_{k} a^{*} a\right)<\infty, \\
\operatorname{Tr}_{\mathscr{F}}\left(\rho_{k}\left|\xi a^{*}+\bar{\xi} a\right|^{2}\right) \leqslant C|\xi|^{2}, \\
\operatorname{Tr}_{\mathscr{F}}\left(\rho_{k}\left|\xi a^{*}+\bar{\xi} a\right|^{3}\right) \leqslant C^{\prime}|\xi|^{3}, \\
\operatorname{Tr}_{\mathscr{F}}\left(\rho_{k}\left|\xi a^{*}+\bar{\xi} a\right|^{4}\right) \leqslant C^{\prime \prime}|\xi|^{4},
\end{gathered}
$$

где $C, C^{\prime}, C^{\prime \prime}$ - положительные постоянные, зависящие только от $\operatorname{Tr}\left(\rho_{1}\left(a^{*} a\right)^{2}\right)$.

ДокАзАтЕльство. Первое ограничение (4.38а) является следствием неравенства Коши-Шварца и гипотезы (h3). Применяя к $A=\bar{\xi}$ a неравенства

$$
\begin{aligned}
\left|A+A^{*}\right|^{2} & \leqslant\left|A+A^{*}\right|^{2}+\left|A-A^{*}\right|^{2}=2\left(A A^{*}+A^{*} A\right), \\
\left|A+A^{*}\right|^{4} & \leqslant\left|A+A^{*}\right|^{4}+\left|A-A^{*}\right|^{4}+\left|A+i A^{*}\right|^{4}+\left|A-i A^{*}\right|^{4}= \\
& =4\left(A A^{*}+A^{*} A\right)^{2}+4\left(A^{2} A^{* 2}+A^{* 2} A^{2}\right),
\end{aligned}
$$

получаем оценки (4.38б) и (4.38г). Наконец, комбинация неравенств (4.38б), (4.38г) с неравенством Коши-Шварца дает оценку (4.38в).

Теорема 4.3. Пусть $\tau \rightarrow 0$ при $N \rightarrow \infty$ есть предел короткого времени взаимодействия при выполнении требований $\tau^{2} N \rightarrow \infty u \tau^{3} N \rightarrow 0$. Тогда для любого начального условия (4.36), удовлетворяющего гипотезам (h1)-(h3), характеристическая функция состояния $\omega_{\mathcal{S}}^{N \tau}(\hat{w}(\theta))$ для подсистемы $\mathcal{S}$ при $t=N \tau$ имеет предел:

$$
\begin{aligned}
\omega_{\mathcal{S}}(\hat{w}(\theta)): & =\lim _{t \rightarrow \infty} \omega_{\mathcal{S}}^{N \tau}(\hat{w}(\theta))= \\
& =\lim _{t \rightarrow \infty} \omega_{\rho(N \tau)}\left(W\left(\zeta_{\theta}\right)\right)=e^{-|\theta|^{2} \operatorname{Tr} \mathscr{F}\left[\rho_{1}\left(a^{*} a+a a^{*}\right)\right] / 4} .
\end{aligned}
$$

Здесъ $\theta \in \mathbb{C} u(N+1)$-компонентный вектор $\left(\zeta_{\theta}\right)^{\mathrm{tr}}:=(\theta, 0,0, \ldots, 0) \in \mathbb{C}^{N+1}$.

ЗАмечАние 4.3. Напомним, что состояние $\omega$ над алгеброй Вейля $\mathscr{A}(\mathscr{F})=\overline{\mathscr{A}_{w}(\mathscr{F})}$ является регулярным, $C^{n}$-гладким или аналитическим, если характеристическая функция состояния $\omega$

$$
s \mapsto \omega(\hat{w}(s \theta))=\omega\left(e^{i s \Phi(\theta)}\right), \quad \Phi(\theta)=\frac{\bar{\theta} a+\theta a^{*}}{\sqrt{2}},
$$

является соответственно непрерывной, $C^{n}$-гладкой или аналитической в окрестности $s=0$. В последнем случае характеристическая функция $\omega(\hat{w}(s \theta))$ (а поэтому и состояние) полностью определяется равенством

$$
\omega(\hat{w}(s \theta))=\exp \left[\sum_{m=1}^{\infty} \frac{i^{m} s^{m}}{m !} \omega_{\mathrm{T}}\left(\Phi^{m}(\theta)\right)\right] .
$$


Здесь $\left\{\omega_{\mathrm{T}}\left(\Phi^{m}(\theta)\right)\right\}_{m=0}^{\infty}-$ усеченные корреляционные функции, задающиеся рекуррентными соотношениями

$$
\omega_{\mathrm{T}}(\Phi(\theta)):=\omega(\Phi(\theta)), \quad \omega_{\mathrm{T}}\left(\Phi^{2}(\theta)\right):=\omega\left(\Phi^{2}(\theta)\right)-\omega(\Phi(\theta))^{2}, \ldots .
$$

Из леммы 4.2 следует, что состояния для матриц плотности $\rho_{1}=\rho_{2}=\cdots$ являются $C^{4}$-гладкими. Заметим, что для калибровочно-инвариантного состояния мы имеем $\omega\left(\Phi^{m}(\theta)\right)=\omega_{\mathrm{T}}\left(\Phi^{m}(\theta)\right)$ (см. гипотезу $\left.(\mathrm{h} 2)\right)$.

ДокАЗАтЕЛЬСтво теоремы 4.3. Согласно (h2) и утверждениям (4.38a)-(4.38в) леммы 4.2 , а также с учетом замечания 4.3 для состояний $\omega(\cdot)=\omega_{\rho_{k}}(\cdot)$ мы можем представить (4.40) в виде

$$
C_{k}(\theta):=\omega_{\rho_{k}}(\hat{w}(\theta))=\exp \left[-\frac{1}{2} \omega_{\rho_{k}}\left(\Phi^{2}(\theta)\right)+R(\theta)\right], \quad k=1,2, \ldots, N,
$$

где $R(\theta)=O\left(|\theta|^{3}\right)$ в окрестности $\theta=0$. Далее, для самосопряженного оператора $\Phi(\theta)=\left(\bar{\theta} a+\theta a^{*}\right) / \sqrt{2}$ с учетом гипотезы (h2) и неравенства (4.38a) из леммы 4.2 мы заключаем, что

$$
\omega_{\rho_{k}}\left(\Phi^{2}(\theta)\right)=\frac{1}{2}|\theta|^{2} \operatorname{Tr} \mathscr{F}\left[\rho_{k}\left(a^{*} a+a a^{*}\right)\right] .
$$

Используя лемму 2.1 для вектора $\left(\zeta_{\theta}\right)^{\operatorname{tr}}:=(\theta, 0,0, \ldots, 0) \in \mathbb{C}^{N+1}$ и соотношения (4.41), (4.42), получаем представление (2.8):

$$
\begin{aligned}
& \omega_{\mathcal{S}}^{N \tau}(\hat{w}(\theta))=\omega_{\rho(N \tau)}\left(W\left(\zeta_{\theta}\right)\right)=C_{0}\left(e^{i \epsilon \tau N}(g z)^{N} \theta\right) \prod_{k=1}^{N} C_{k}\left(e^{i \epsilon \tau N} g w(g z)^{N-k} \theta\right)= \\
& =\omega_{\rho_{0}}\left(\hat{w}\left(\theta_{0}(\tau)\right)\right) \exp \left[-\sum_{k=1}^{N} \frac{\left|\theta_{k}(\tau)\right|^{2}}{4} \operatorname{Tr}_{\mathscr{F}}\left[\left(a^{*} a+a a^{*}\right) \rho_{k}\right]+\widehat{R}\right] .
\end{aligned}
$$

Здесь $\theta_{0}(\tau)$ и $\theta_{k}(\tau)$ определяются соотношениями $(2.19)$ и $(2.20)$ при $\theta \equiv \zeta_{0}$ :

$$
\begin{aligned}
& \theta_{0}(\tau)=\left(U_{1}(\tau) \ldots U_{N}(\tau) \zeta_{\theta}\right)_{0}=e^{i \epsilon \tau N}(g z)^{N} \theta \\
& \theta_{k}(\tau)=\left(U_{1}(\tau) \ldots U_{N}(\tau) \zeta_{\theta}\right)_{k}=e^{i \epsilon N \tau} g w(g z)^{N-k} \theta
\end{aligned}
$$

при этом $\widehat{R}=\sum_{k=1}^{N} O\left(\left|\theta_{k}\right|^{3}\right)$ согласно формуле (4.41).

Напомним, что в силу соглашения (1.22) (см. замечание 1.2 ) мы имеем $g=g(\tau)$, $w=w(\tau), z=z(\tau)$. Тогда с учетом представлений (1.12), (1.13) получаем $|g(\tau)|=1$, $|w(\tau)|^{2}+|z(\tau)|^{2}=1$ и, кроме того, при малых $\tau$

$$
w(\tau)=i \eta \tau+O\left(\tau^{3}\right), \quad|z(\tau)|=1-\frac{\eta^{2} \tau^{2}}{2}+O\left(\tau^{4}\right) .
$$

Следовательно, при $\tau \rightarrow 0$ и $\tau^{2} N \rightarrow \infty$ это дает оценки

$$
\left|(g(\tau) z(\tau))^{N}\right| \leqslant O\left(e^{-\eta^{2} \tau^{2} N / 2}\right), \quad\left|\theta_{k}\right| \leqslant O(\tau), \quad \widehat{R}=O\left(\tau^{3} N\right)
$$

для любого фиксированного параметра взаимодействия $\eta$.

Согласно замечанию $4.3 \omega_{\rho_{0}}(\hat{w}(\alpha))$ является характеристической функцией регулярного состояния. Поэтому она непрерывна в точке $\alpha=0$ и $\omega_{\rho_{0}}(\hat{w}(0))=1$. Отметим 
также, что согласно гипотезе (h1), касающейся матриц $\left\{\rho_{k}\right\}_{k=1}^{N}$, для оценки (4.43) нам нужно контролировать сумму

$$
\sum_{k=1}^{N}\left|\theta_{k}(\tau)\right|^{2}=|\theta|^{2}|w(\tau)|^{2} \frac{1-|z(\tau)|^{2 N}}{1-|z(\tau)|^{2}} .
$$

С учетом условий $\tau^{2} N \rightarrow \infty$ и $\tau^{3} N \rightarrow 0$ получаем пределы

$$
\begin{gathered}
\lim _{\tau \rightarrow 0, N \rightarrow \infty} \omega_{\rho_{0}}\left(\hat{w}\left(\theta_{0}(\tau)\right)\right)=1, \\
\lim _{\tau \rightarrow 0, N \rightarrow \infty} \sum_{k=1}^{N}\left|\theta_{k}(\tau)\right|^{2}=|\theta|^{2}, \quad \lim _{\tau \rightarrow 0, N \rightarrow \infty} \widehat{R}=0 .
\end{gathered}
$$

Подставляя их в (4.43), выводим утверждение (4.39). Теорема доказана.

ЗАмЕчАниЕ 4.4. Напомним, что из предельных соотношений (4.39) для характеристических функций следует, что регулярные состояния $\omega_{\mathcal{S}}^{N \tau}$ сходятся к регулярному стационарному состоянию $\omega_{\mathcal{S}}$ в слабой* топологии (см. замечание 2.2). Из правой части выражения (4.39) с учетом определения 3.1 следует, что предельное состояние является калибровочно-инвариантным и квазисвободным, если форма $h(\theta):=|\theta|^{2} \operatorname{Tr}_{\mathscr{F}}\left(\rho_{1} a^{*} a\right)$ задана в $(3.2)$.

СлеДСТвИЕ 4.1. Предположим, что матрицы плотности $\left\{\rho_{k}\right\}_{k=1}^{N}$ coответствуют калибровочно-инвариантному квазисвободному гиббсовскому состоянию с параметром $\beta$ (3.20). Эти состояния удовлетворяют гипотезам (h1)-(h3). Тогда, проводя явные вычисления в (4.39), находим, что утверждение теоремы 4.3 справедливо в пределе

$$
\lim _{\tau \rightarrow 0, N \rightarrow \infty} \omega_{\mathcal{S}}^{N \tau}(\hat{w}(\theta))=\exp \left[-\frac{|\theta|^{2}}{4} \frac{1+e^{-\beta}}{1-e^{-\beta}}\right] .
$$

Эта характеристическая функиия совпадает с (4.6) для гиббсовского равновесного состояния подсистемы $\mathcal{S}$ с параметром $\beta$, определяемым бесконечной иепочкой атомов.

Уместны несколько замечаний.

ЗАмЕчАниЕ 4.5. Теорема 4.3 описывает эволюцию подсистемы $\mathcal{S}$ в пределе кратковременного повторяющегося взаимодействия (при $\tau \rightarrow 0$ ) и при условии, что параметр времени $t=\tau N \rightarrow \infty$ для фиксированной постоянной взаимодействия $\eta$. В таком пределе непрерывного времени для бесконечной цепочки атомов, которые все находятся в гиббсовском состоянии с температурным параметром $\beta$, получаем то же стационарное состояние (4.44) системы $\mathcal{S}$, что и для дискретного времени $\tau>0$ (см. соотношение (4.6)). Хотя, возможно, это не выглядит неожиданным, мы хотели бы подчеркнуть, что для нашего варианта предельного перехода к непрерывному времени мы можем ослабить условия на начальное состояние-произведение (4.36) для системы $\mathcal{S}+\mathcal{C}$ до гипотез (h1)-(h3) вместо частного выбора (3.20).

ЗАмЕЧАНИЕ 4.6. Еще один, более сложный, предел непрерывного времени $\tau \rightarrow 0$, $t=\tau N<\infty$ впервые рассматривался в контексте повторяющегося возмущения 
в работе [11]. Показано, что для некоторых моделей этот предел аналогичен некоммутативной центральной предельной теореме [7], для чего требуется подходящий скейлинг постоянной взаимодействия $\eta$. Тогда цепочка $\mathcal{C}$ действует как внешний резервуар квантовых шумов. Такая концепция квантовых шумов была обобщена в работе [12], что позволило в явном виде получить генератор Линдбланда для эффективной марковской динамики на $\mathcal{S}$ (см. также работу [13]).

ЗАмЕчАниЕ 4.7. Из доказательства теоремы 4.3 следует также, что начальное состояние $\omega_{\mathcal{S}}$ подсистемы $\mathcal{S}$ можно заменить на любое регулярное состояние.

Благодарности. Мы хотели бы поблагодарить рецензента за полезные замечания и предложения. В.А. Загребнов выражает благодарность Institute of Science and Engineering, Graduate School of the Natural Science and Technology (Kanazawa University, Japan) за поддержку и гостеприимство.

\section{Список литературы}

[1] H. Tamura, V. A. Zagrebnov, A dynamics driven by repeated harmonic perturbations, arXiv: 1404.2998.

[2] B. Nachtergaele, A. Vershynina, V.A. Zagrebnov, Ann. Henri Poincaré, 15:2 (2014), 213-262, arXiv: 1206.6169.

[3] L. Bruneau, A. Joye, M. Merkli, J. Math. Phys., 55:7 (2014), 075204, 67 pp., arXiv: 1305.2472.

[4] O. Bratteli, D. W. Robinson, Operator Algebras and Quantum Statistical Mechanics 2. Equilibrium States. Models in Quantum Statistical Mechanics, Springer, Berlin, 1997.

[5] V.A. Zagrebnov, Topics in the Theory of Gibbs Semigroups, Leuven Notes in Mathematical and Theoretical Physics. Ser. A: Mathematical Physics, 10, KU Leuven Univ. Press, Leuven, 2003.

[6] S. Attal, A. Joye, C.-A. Pillet (eds.), Open Quantum Systems I. The Hamiltonian Approach (Grenoble, France June 16 - July 4, 2003), Lecture Notes in Mathematics, 1880, Springer, Berlin, 2006.

[7] A. F. Verbeure, Many-Body Boson Systems. Half a Century Later, Springer, Berlin, 2011.

[8] M. Fannes, Ann. Inst. Henri Poincaré Sect. A (N. S.), 28:2 (1978), 187-196.

[9] H. Araki, Publ. Res. Inst. Math. Sci., 11:3 (1976), 809-833.

[10] S. Attal, A. Joye, C.-A. Pillet (eds.), Open Quantum Systems III. Recent Developements (Grenoble, France June 16-July 4, 2003), Lecture Notes in Mathematics, 1882, Springer, Berlin, 2006.

[11] S. Attal, Y. Pautrat, Ann. Inst. Henri Poincaré, 7:1 (2006), 59-104.

[12] S. Attal, A. Joye, C.-A. Pillet (eds.), Open Quantum Systems II. The Markovian Approach (Grenoble, France June 16 - July 4, 2003), Lecture Notes in Mathematics, 1881, Springer, Berlin, 2006.

[13] S. Attal, C. Pellegrini, Open Syst. Inf. Dyn., 17:4 (2010), 389-408.

Поступила в редакцию 13.07.2015, после доработки 20.10.2015 